\title{
HISTORIA DE LAS DEMANDAS Y OTRAS ACCIONES LEGALES ENTABLADAS CONTRA LA INDUSTRIA TABAQUERA EN LOS ESTADOS UNIDOS
}

\author{
Goretti Vadillo Robredo \\ Doctor en Derecho
}

Sumario: I. Introducción. II. Demandas de particulares contra la industria tabaquera. 1. Introducción. 2. Antes de Cipollone. 3. Cipollone v. Liggett Group Inc. a. En general. b. En concreto. 4. Después de Cipollone. 5. Fumadores pasivos. II. «Class Action» v. Tabacaleras. 1. Castano v. American Tobacco Co. 2. Broin v. Philip Morris. 3. Engle v. R.J. Reynolds Tobacco, et.al. III. Demandas entabladas por los estados (Attorneys General). IV. Acuerdos extrajudiciales. Comentario sobre el preacuerdo de junio de 1997. Preacuerdo de 23 de noviembre de 1998: Multistate Master Settlement Agreement (MSA). V. Recapitulación.

\section{Introducción}

La responsabilidad por productos defectuosos es uno de los temas jurídicos que más atención acapara, tanto por parte de los medios de comunicación no especializados como de la doctrina y la jurisprudencia de los distintos países. En los Estados Unidos — país en el que más atención se ha dedicado a este tema- las distintas épocas de la evolución de la responsabilidad por productos defectuosos han estado marcadas por algunos productos en particular. Productos como el asbesto, el Dalkon Shield, el DES, el Ford Pinto y las consecuencias dañosas derivadas de su utilización han determinado, en gran medida, que la doctrina y la jurisprudencia norteamericana hayan evolucionado hasta la situación actual. Muchos de los casos más conocidos son supuestos de accidentes en masa (mass tort). En estos casos, la utilización o el consumo de un mismo producto defectuoso dentro de una comunidad más o menos amplia de consumidores provoca un gran número de daños de 
entidad diversa. Los problemas que plantean estas situaciones son complejos. Por un lado, el demandante se enfrenta, entre otros, a serios problemas de prueba de la relación de causalidad. Por otra parte, el demandado, si es declarado responsable, deberá afrontar indemnizaciones millonarias que le pueden llevar a una declaración de quiebra. A simple vista, el sistema clásico de responsabilidad civil parece fracasar en estos casos. Si se busca la indemnización de la víctima, ésta puede verse frustrada con la declaración de quiebra. Por ello, la solución de muchos de estos supuestos ha llevado a la doctrina y a la jurisprudencia norteamericana a elaborar nuevas teorías (por ejemplo, la teoría de «market share») y a buscar nuevas soluciones.

El tabaco constituye un buen ejemplo de la evolución de la responsabilidad por productos defectuosos en los Estados Unidos, evolución que no nos permite considerar que el problema esté zanjado.

Los Estados Unidos son el mayor fabricante de cigarrillos del mundo. Allí se encuentran asentadas las empresas tabacaleras más importantes. Se estima que el impacto de la industria del tabaco en la economía norteamericana fue, en 1994, de unos 54,3 billones de dólares, y que para su cultivo se dedicaban unas 124.000 granjas en 21 estados y Puerto Rico, en las que trabajaron unas 142.000 personas a tiempo completo. En la venta y distribución de tabaco trabajaron, también en 1994, unas 99.000 personas, y en su fabricación unas 42.000. La otra cara de esta saneada industria la componen las 400.000 personas que mueren anualmente en los Estados Unidos por alguna causa atribuible al tabaco.

A finales de la década de los cincuenta comienza, de forma aislada, lo que más tarde sería conocido como la «guerra del tabaco», que ha llegado hasta nuestros días. Las primeras demandas contra las tabacaleras, entabladas por particulares, se encontraron con una incipiente teoría de responsabilidad por productos defectuosos y fracasaron en lograr una indemnización. Este movimiento continuó hasta la década de los noventa. El número de las demandas contra las tabacaleras crecía en todos los estados. Sin embargo, las defensas que planteaba la industria, de forma uniforme, tenían más éxito ante los tribunales que las alegaciones de los demandantes. A pesar de ello, el movimiento continuó, y ha sido en la década de los noventa cuando los demandantes han tenido más éxito en los tribunales. En los noventa, las causas de pedir de los demandantes ya no se limitan a la responsabilidad por productos defectuosos u otras similares, sino que a las alegaciones tradicionales se han añadido las de conspiración, fraude, etc. También es en los noventa cuando surgen las primeras class actions y las demandas entabladas por los Attorney General en reclamación de los costes sanitarios causados 
por enfermedades relacionadas con el consumo de tabaco, además aparecen los fumadores pasivos como nueva categoría de demandantes y la adición a la nicotina como nuevo daño.

Con este panorama, dibujado a grandes rasgos, se puede intuir que la situación para las tabacaleras norteamericanas ha cambiado. El grupo Liggett fue el primero en abandonar el frente común, que la industria del tabaco había constituido para afrontar su defensa, y negociar con los estados. Sería el primer paso de una negociación que más tarde (20 de junio de 1997) se llevaría a cabo entre toda la industria tabaquera y los Attorneys General de 40 estados. El acuerdo resultante de la negociación debía ser ratificado en el Congreso de los Estados Unidos y convertido en ley federal. Sin embargo, nunca se llegó a un acuerdo final en el Congreso.

En el presente artículo $^{1}$, haremos un recorrido por la historia legal de lo que, vulgarmente, se ha conocido como la «guerra del tabaco», analizando los leading cases que marcaron un nuevo paso o movimiento. Finalmente, examinaremos la situación actual en los Estados Unidos.

\section{Demandas de particulares contra la industria tabaquera}

\section{Introducción}

Los primeros en entablar demandas en contra de las tabacaleras ${ }^{2}$ fueron los particulares. Las demandas comenzaron en la década de los cincuenta, e inicialmente se basaban en la teoría de negligence, aunque sin éxito para los demandantes ${ }^{3}$. Los problemas a los que se enfrentaban los particulares eran muy similares. En un primer momento, fueron incapaces de demostrar la relación de causalidad entre el daño (la

${ }^{1}$ El artículo actual tiene su origen en otro anterior de la autora, Situación legal de la industria del tabaco en Estados Unidos, Rev del Colegio de Abogados del Señorío de Vizcaya, 1997, n. ${ }^{\circ}$ 6, pp. 20 y ss. En el momento de elaboración del primer artículo el acuerdo alcanzado entre los estados y las tabacaleras no había sido aprobado. En el presente artículo se examina el acuerdo y las críticas que ha recibido por sectores importantes tanto de la doctrina jurídica como de la económica. Asimismo, se profundiza en materias que, por falta de espacio, no fueron tratadas en el anterior y se prescinde de otras.

${ }^{2}$ En el presente artículo utilizaremos frecuentemente el término «tabacaleras». Con este término queremos hacer referencia a todas las empresas fabricantes de productos relacionados con el tabaco (puros, cigarrillos, etc.). En otras ocasiones utilizaremos expresiones como «industria tabaquera», «industria de tabaco» con el mismo significado.

3 Ver, Rabin, L.R., A Sociolegal History of the Tobacco Tort Litigation, Stanford Law Review, 1992, vol. 44, pp. 853-854. 
muerte o las lesiones del demandante) y la acción u omisión del demandado ${ }^{4}$. Si bien en los años cincuenta ya existían estudios científicos que apuntaban a la relación entre el consumo del tabaco y el cáncer de pulmón ${ }^{5}$, éstos eran discutidos por un sector de la comunidad científica. Ante la falta de certeza, los tribunales optaron por considerar que no se había probado la relación de causalidad entre ambos extremos. En la década de los sesenta se recoge en el Restatement (Second) of Torts la responsabilidad objetiva de los fabricantes de productos defectuosos (sec. $402 \mathrm{~A})^{6}$. La nueva teoría de responsabilidad provocó un giro en el planteamiento de las demandas contra las tabacaleras. Es en esta época cuando las demandas se basan en la strict liability y concretamente en el «defecto de información»; es decir, en que las empresas fabricantes de tabaco fracasaron en informar adecuadamente a los fumadores sobre los peligros asociados con su consumo.

La defensa de las tabacaleras también ha sido, más o menos, uniforme. Las defensas comprenden desde la alegación de falta de relación de causalidad, la asunción de riesgo por parte de la víctima, la compensación de culpas y el que la acción está «preempted» ${ }^{7}$.

4 En 1954, Eva Cooper demandó a R.J. Reynolds Tobacco Co. por la muerte de su marido como consecuencia de un cáncer de pulmón. El tribunal decidió que no existía prueba de que el fumar causase cáncer.

5 El 2 de mayo de 1950, Levin, M., publicó en JMA el primer estudio que relacionaba el fumar con el cáncer de pulmón, Tobacco Smoking as a Possible Etiologic Factor in Bronchiogenic Carcionoma: A Study of 684 Proved Cases. El 30 de setiembre de 1950, el British Medical Journal publicó un estubio de Doll, R. y HiLl, B., en el que se afirmaba que los fumadores con un nivel alto de consumo tenían un $50 \%$ más de probabilidad que los no fumadores de contraer cáncer de pulmón.

6 The Restatement of Torts (Second), 402 A (1965):

Special Liability of Seller of Product for Physical Harm to User or Consumer

(1) One who sells any product ina defective condition unreasonably dangerous to the user or consumer or to his property is subject to liability for physical harm, thereby causad to the ultimate user or consumer, or to his property, if

(a) the seller is engaged in the business of selling such a product, and

(b) it is expected to and does reach the user or consumer without substantial change in the condition in which it is sold.

(2) The rule stated in Subsection (1) applies althogh

(a) the seller has exercised all possible care in the preparation and sale of his product, and

(b) the user or consumer has not bought the product from or entered into any contractual relation with the seller.

7 BLACK'S LAW DICTIONARY, p. 815, «Preemption: Doctrine adopted by the U.S. Supreme Court holding that certain matters are of such a national, as opposed to local, character that federal laws preempt or take precedence over state laws. As such, a state may not pass a law inconsistent with the federal law. Examples are federal laws governing interstate commerce». p. 424, «Federal pre-emption: The U.S. Constitution and acts of Coogress have 
A pesar de la falta de éxito logrado por las demandas entabladas de forma individual, la interposición de demandas contra la industria tabaquera no ha cesado. Ante la negativa de los tribunales de conceder indemnización, los abogados de ambas partes han recurrido a nuevas teorías para apoyar sus alegaciones. Por ejemplo, en el inicio de lo que se ha conocido como la «guerra del tabaco», el daño alegado por las víctimas era, casi de forma exclusiva, enfermedades pulmonares y de corazón. A finales de la década de los ochenta, se incorpora como daño la adición a la nicotina. Además, las víctimas presuntas ya no son sólo los fumadores directos, sino que también se incorporan a las demandas los fumadores pasivos - por los daños sufridos como consecuencia de la inhalación del humo que se desprende del consumo de tabaco-

El caso líder es Cipollone v. Liggett Group $\mathrm{Inc}^{8}$. A continuación analizaremos la situación anterior al caso Cipollone, el caso Cipollone, y las modificaciones posteriores como consecuencia de esta sentencia.

\section{Antes de Cipollone}

Las primeras acciones contra las tabacaleras se basaron en la alegación de incumplimiento de garantías implícitas o explícitas. Se alegaba, principalmente, que la industria del tabaco, por medio de su publici$\mathrm{dad}^{9}$, había garantizado expresamente a sus consumidores que su producto era seguro y esta garantía se había incumplido.

Una demanda por incumplimiento de garantía debe probar 5 elementos $^{10}$ :

1. La existencia de la garantía;

2. el hecho de que el estado de los bienes fuese diferente del establecido en la garantía;

3. que la lesión fue el resultado del incumplimiento de la garantía;

4. el quantum indemnizatorio;

5. que los hechos relacionados con los daños sufridos por incumplimiento de la garantía superan las defensas alegadas por el demandado.

given to the federal government exclusive power over certain matters such a as interstate commerce and sedition to the exclusion of state jurisdiction. Occurs where federal law so occupies the field that state courts are preventes from asserting jurisdiction».

8112 S.Ct. 2608, 120 L.De.2d 407 (1992).

9 Por ejemplo, el grupo Liggett decía «Usted no puede comprar un cigarrillo mejor».

10 Art. 2 del Uniform Commercial Code. 
El fracaso de estas primeras demandas tuvo su causa en que la acción por incumplimiento de garantía fue creada para regular los tratos comerciales entre las partes, y el plazo para entablar la demanda es de 4 años desde que se entregó el producto. En la gran mayoría de las demandas entabladas contra las tabacaleras, el plazo había expirado mucho antes de que el demandante fuese consciente del daño o de la lesión. A pesar del fracaso continuo de la demanda por incumplimiento de garantía en los tribunales norteamericanos, ésta se mantiene en las acciones presentes contra la industria del tabaco.

La segunda teoría invocada por los demandantes ha sido la de $n e-$ gligence. «Negligence» es el término utilizado para describir la conducta del demandado. Pero una acción basada en «negligence» requiere algo más que una conducta negligente o culpable. Debe haber una obligación, y debe haber consecuencias derivadas del incumplimiento de dicha obligación. La fórmula tradicional de esta causa de acción incluye los siguientes elementos:

1. La obligación de utilizar un cuidado razonable.

2. El fracaso de cumplir con el patrón requerido.

3. La relación de causalidad entre la conducta y la lesión resultante, comúnmente llamada «proximate cause» y algunas veces «legal cause».

4. Pérdida o daño real.

El fracaso de estas acciones en los tribunales se debió, principalmente, a la falta de prueba de la relación de causalidad ${ }^{11}$.

La tercera teoría esgrimida contra las tabacaleras era la teoría de strict liability — basada en la sección 402 A del Restatement (Second) of Torts - en la que se establece la responsabilidad objetiva por el daño resultante de un producto «unreasonably dangerous». La industria del tabaco se amparaba en las defensas basadas en la conducta de la víctima (compensación de culpas y asunción del riesgo por la víctima). La responsabilidad objetiva recogida en el Restatement y aplicada por los tribunales norteamericanos no supone una responsabilidad absoluta. Al igual que en el Derecho continental, los demandantes norteamericanos tienen

11 Ver entre otros, Hudson v. R.J. Reynolds Tobacco Co., 427 F.2d 541 (5 ${ }^{\text {th }}$ Cir. 1970); Green v. American Tobacco Co., 409 F.2d 1166 (5 $5^{\text {th }}$ Cir. 1969), cert. Denied, 386 U.S. 1009 (1967); Ross v. Philip Morris \& Co., 328 F.2d 3 ( $8^{\text {th }}$ Cir. 1964); Lartigue v. R.J. Reynolds Tobacco Co., 317 F.2d 19 (5 ${ }^{\text {th }}$ Cir.), cert. Denied, 375 U.S. 865 (1963); Cooper v. R.J. Reynolds Tobacco Co., 256 F.2d 464 (1 $1^{\text {st }}$ Cir. 1958); Albright v. R.J. Reynolds Tobacco Co., 350 F. Supp. 341 (W.D.Pa. 1972). 
que probar la existencia del defecto, el daño sufrido por la víctima en su persona o en su propiedad y la relación de causalidad entre ambos. Además se admiten defensas, muchas de ellas basadas en la propia conducta de la víctima ${ }^{12}$, existentes también en la teoría de negligence.

Las defensas fundadas en la conducta de la víctima fueron la clave del éxito de las tabacaleras en los tribunales, si bien, análisis posteriores cuestionan la viabilidad de las defensas. La Sección 402 A del Restatement, en el comment «n», recoge que la conducta de la víctima puede constituir una defensa afirmativa cuando ella «voluntarily and unreasonably proceed(s) to encounter a known danger» es la defensa que se conoce como «asunción de riesgo» ${ }^{13}$. La defensa, en teoría, exi-

12 El elemento central en el análisis de responsabilidad objetiva, al haberse eliminado la actuación culpable o negligente del agente ilícito, es el defecto del producto. Los distintos defectos alegados en las demandas contra las Tabacaleras serán analizados en el siguiente apartado cuando se comente el leading case Cipollone. Vid. FISCHER, D.A., PoWERS, Jr. W., Products Liability. Cases and Materiales, 2. ${ }^{a}$ edic., St. Paul, Minn., West Publishing Co., 1994.

${ }^{13}$ Hildebrand v. Minyard (Arizona Court of Appeals, 1972. 16 Ariz. App. 538, 494 P.2d 1328):

«There are two types of assumption of risk, express assumption of risk and implied assumption of risk. In the express assumption of risk situation the plaintiff expressly agrees in advance that the defendant is under no obligation to care for him and shall not be liable for the consequences of conduct which would otherwise be negligent.

Implied assumption of risk requires the presence of the following elements:

(1) There must be a risk of harm to plaintiff caused by defendant's conduct or by the condition of the defendant's land or chaffels;

(2) Plaintiff must have actual knowledge of the particular risk and appreciate its magnitude.

(3) The plaintiff must voluntarily choose to enter or remain within the ares of the risk under circumstances that manifest his willingness to accept that particular risk.

As with express assumption of risk, the touchstone of implied assumption of risk is "consent" ".

La asunción de riesgo y la compensación de culpas han sido confundidas en ocasiones. Los demandantes buscan la calificación de la conducta de la víctima como aquella constitutiva de compensación de culpas ya que ésta, en la gran mayoría de los estados, no supone una defensa total sino una reducción del quantum indemnizatorio. Por el contrario, los demandados buscan la calificación de la conducta como asunción de riesgo, ya que ésta sí supone una defensa total y el fracaso total de la acción del demandante. Prosser, W.L., Torts, 7. a edic, New York, Foundation Press, 1982, p. 445:

"It is here that there is the greatest misapprehension and confusion as to assumption of risk, and its most frequent misapplication. It is not true that in any case where the plaintiff voluntarily encounters a known danger he necessarily consents to negligence of the defendant which creates it. A pedestrian who wlaks across me street in me middle of a block, through a stream of traffic travelling at 
ge que la víctima sea competente para conocer la condición peligrosa del producto, conocer que la actividad en cuestión es peligrosa, apreciar la naturaleza y extensión del peligro y que voluntariamente se exponga al riesgo. Sin embargo, los tribunales norteamericanos se encuentran divididos sobre esta cuestión. Algunos tribunales han requerido que se pruebe la competencia de la víctima para conocer la magnitud del riesgo, mientras que otros, más favorables a los demandados, han considerado suficiente la prueba de un conocimiento general del riesgo ${ }^{14}$.

La quiebra de la defensa de asunción del riesgo por la víctima se ha buscado en tres campos: la competencia de la víctima para valorar los riesgos del tabaco, el conocimiento de la víctima de los riesgos del tabaco, y la voluntariedad de la víctima en el consumo de tabaco ${ }^{15}$.

Los demandantes que comenzaron a fumar siendo menores han planteado la cuestión de su propia competencia para valorar los riesgos del consumo de tabaco, argumentando que no eran legalmente capaces para ello ${ }^{16}$. Algunos autores han argüido que los menores deberían ser considerados incompetentes per se para asumir los riesgos del tabaco - de la misma forma que lo son para consentir en las relaciones sexuales o para las relaciones contractuales ${ }^{17}$. Otra posibilidad es que no se considere a todos los menores incapaces para valorar los riesgos del tabaco, sino que se analice cada caso de forma individual. Esta última postura parece haber sido la adoptada por un mayor número de tribunales ${ }^{18}$.

high speed, cannot by any stretch of the imagination be found to consent that the drivers shall not use care to avoid running him down. On the contrary, he is insisting that me shall. This is contributory negligence, pure and simple; it is not assumption of risk».

Ver también, Restatement (Second) of Torts 496 f C ) (1971), comment h.

${ }^{14}$ Una prueba sustancial de la competencia de la víctima para conocer el tipo y la magnitud específica del riesgo se exigió en Gryc v. Dayton-Hudson Corp., 297 N.W. 2d 727, 743 (Minn. 1980). En Toetschinger v. Ihnot, 312 Minn. 59 69-70, 250 N.W. 2d (1977) se aceptó un conocimiento general del riesgo como suficiente.

15 Para un análisis en profundidad de estas cuestiones ver NOTE, Plaintiff's conduct as a defense to claims against cigarette manufacturers, Harvard Law Review, 1986, vol. 99, pp. 809-827.

16 Según un estudio del U.S. DEP'T OF HEALTH, EDUC. and WELFARE, Office on Smoking and Health, U.S. Dep't of Health, Educ. And Welfare, Smoking and Health: A Report of the Surgeon General, 1979, la gran mayoría de los fumadores adultos comenzaron a fumar antes de alcanzar la edad de 21 años. Recogido en Plaintiff's conduct as a defense, loc.cit, p. 812.

17 Por ejemplo, WHITE, Strict Liability of Cigarette Manufacturers and Assumption of Risk, 29 La.L.Rev., 1969, vol. 29, pp. 589 y ss.

18 En la NOTE, Plaintiff's conduct as a defense, loc.cit, p. 816 se relaciona la incompetencia de los menores con la naturaleza adictiva del tabaco. Según la NOTE, si los menores 
Los demandados alegaron que la víctima actuaba con conocimiento del riesgo al que se enfrentaba. Los demandados deben probar que el peligro era tan obvio y notorio que se puede presumir su conocimiento o que el fabricante explícitamente informó al usuario del peligro del producto $^{19}$. Es cierto que existen pocos productos en el mercado sobre los que se conozcan los riesgos de su utilización tan bien como los del consumo de tabaco. Además, desde 1965 los paquetes de tabaco contienen un aviso explícito sobre los peligros del fumar. Como resultado, se ha defendido que los fumadores de forma consciente asumen los riesgos del tabaco. Algunos autores y demandantes han respondido que la industria no puede establecer que los fumadores conocen «suficientemente» los peligros del tabaco, ya que el conocimiento del público no se extiende a enfermedades particulares relacionadas con el consumo de dicho producto (como, por ejemplo, las enfermedades del corazón o los problemas durante el embarazo). Es decir, que el público no posee un conocimiento específico del riesgo al que se enfrenta. Además, se dice que los fumadores no han sido conscientes del riesgo a la adición que conlleva el uso de tabaco. Este último argumento es el más utilizado en la actualidad. Sorprende que mientras que la industria ha negado de forma insistente la relación de causalidad entre el consumo de tabaco y las enfermedades de cáncer de pulmón, y otras, se alegue luego como uno de los conocimientos más comunes entre la población.

La adicción provocada por la nicotina ha sido utilizada como herramienta para negar la voluntariedad en el consumo del tabaco ${ }^{20}$. Doctrinalmente, un demandante adicto a un producto (en el que no se informase de su naturaleza adictiva) no puede considerarse que haya asumido el riesgo ${ }^{21}$. Sin embargo, la naturaleza adictiva no sirve como

son declarados incompetentes para valorar los riesgos del consumo del tabaco y por lo tanto no responsables legalmente por su decisión de fumar, los demandados podrían alegar que su decisión de continuar fumando en la edad adulta ratifica la decisión anterior. Sin embargo, si el demandante consigue probar que fumar es adictivo y que adquirió esa adición antes de ser competente para valorar los riesgos del tabaco, el demandado será incapaz de probar que la decisión de continuar fumando en la edad adulta ratificaba la voluntariedad en el consumo durante la edad no adulta.

19 Restatement (Second) of Torts 402 A comment $j$.

20 Aunque la capacidad de millones de fumadores para dejar de fumar cuestionó la naturaleza adictiva de la nicotina, hoy existe consenso dentro de la comunidad médica de que el fumar es adictivo.

21 Uno de los pocos casos que ha tratado esta cuestión ha sido Crocker v. Winthrop Laboratories, 502 S.W. 2d 850 (Tex.Civ.App. 1973). En este caso, se consideró que el demandado no había sido contributivamente negligente en la causación de su propia muerte al tomar el narcótico Talwin, porque esta acción era resultado de su adicción a dicho narcótico. Sentencia recogida en Plaintiff's conduct as a defense, loc.cit, p. 816. 
respuesta a la defensa de asunción de riesgo si el demandado había sido informado de ella. La información contenida en las cajetillas de tabaco en los Estados Unidos no informa sobre la naturaleza adictiva del taba$\mathrm{co}^{22}$, por lo que, en principio - y teniendo en cuenta que sólo en 1998 han reconocido los ejecutivos de las empresas tabacaleras que la nicotina es adictiva - no se puede llegar a la conclusión de que los consumidores estuviesen informados.

En conclusión, la alegación de la asunción del riesgo por parte de la víctima como defensa absoluta no parece ser una defensa tan contundente como pudiera parecer a simple vista, y ha dado lugar a opiniones discrepantes en los tribunales dependiendo del grado de conocimiento que éstos exijan.

\section{Cipollone v. Liggett Group Inc. ${ }^{23}$}

Cipollone representa el caso típico de la demanda de un particular contra una tabacalera. Su importancia reside no en el supuesto de hecho, sino en que en esta decisión el Tribunal contestó a preguntas que se estaban planteando en distintas jurisdicciones y para las que parecía no haber una solución uniforme. A partir del caso Cipollone, las estrategias de los demandantes y de los demandados dieron un nuevo giro, que es, en cierta medida, el que ha llegado hasta la actualidad.

Antonio Cipollone pide que se declare responsable al grupo Liggett (y a otros) por la muerte de su mujer como consecuencia de un cáncer de pulmón ${ }^{24}$. En la demanda se alegaba que el grupo Liggett había fracasado en informar sobre los peligros del consumo de tabaco (defecto de información en el producto), que la Compañía había incumplido la garantía expresa (express product warranty), que el producto poseía un defecto de diseño porque la empresa fracasó en comercializar la versión más segura del producto, y que existió una conspiración - coordinada entre toda la industria tabacalera - para defraudar y confundir al público sobre los peligros del tabaco.

Liggett, en su defensa, alega que la acción entablada por Cipollone está preempted, como consecuencia de la Federal Cigarette Labeling

22 En 1965, el Congreso aprobó que en todas las cajetillas de tabaco se recogiese la advertencia: «Caution-cigarette smoking may be hazardous to your health». En $1970 \mathrm{y}$ 1084 se modificó la advertencia.

23112 S.Ct. 2608, 120 L.De.2d 407 (1992).

24 Rose Cipollone nació en 1925 y comenzó a fumar en 1942. Fumó Chesterfield hasta 1955, cuando cambió a la marca L \& M. Desde 1942 a 1980 Rose fumó una media de dos paquetes diarios. En 1981 se le diagnosticó un cáncer de pulmón y en 1983 dejó de fumar. Para entonces su enfermedad era terminal. Fallece en 1984. 
and Advertising Act de 196525. Asimismo, alega que el comportamiento de Rose Cipollone supone un caso claro de asunción de riesgo por la víctima, o en su defecto, de culpa contributiva de la víctima. El grupo Liggett rechaza también cualquier alegación de que su producto tuviese un defecto de información o un defecto de diseño.

El jurado, después de un juicio de cuatro meses, emitió un veredicto a favor de Antonio Cipollone por incumplimiento de Liggett de express warranty, en el que se le concedía una indemnización de 400.000 dólares. El jurado también declaró que el demandado era responsable por defecto de información sobre los peligros de su producto, pero emitió un veredicto a favor de Liggett por considerar que Rose Cipollone también había sido culpable. El jurado especificó que Rose era responsable del $80 \%$ de sus lesiones, porque conoció y apreció el daño del consumo de cigarrillos, y, aún así, voluntariamente eligió fumar. El District Court dijo que las demandas basadas en defecto de información estaban preempted por la Federal Labelling and Advertising Act de 1965 y 1969. La Court of Appeals afirmó este punto26. Los componentes del Tribunal Supremo estuvieron divididos sobre esta cuestión. Tres de los nueve magistrados pensaban que ninguna de las acciones estaba preempted. Los otros seis discrepaban en el grado.

\section{a. En general}

En principio, la demanda entablada por Antonio Cipollone no plantea peculiaridades frente a las demandas entabladas en el pasado. El defecto de diseño ${ }^{27}$ se había alegado en las demandas contra las tabacaleras pero no había tenido éxito, ya que no se pudo probar que existiese un diseño razonable alternativo. La totalidad de las demandas entabladas por los particulares se han basado, al menos en parte, en el «defec-

25 Reformada y recogida en 15 U.S.C. 1331-1340 (1982 \& Supp. II 1984).

26 United States Court of Appeals, Third Circuit, 1990. 893 F.2d 541.

27 Un producto puede ser irrazonablemente peligroso por varias razones. En primer lugar, el defecto puede estar en el diseño, y en este caso toda la línea de productos será «irrazonablemente peligrosa». El demandante debe ofrecer un diseño alternativo y razonable que el fabricante podía haber utilizado. El diseño alternativo debería haber prevenido la lesión y no reducir substancialmente la efectividad del producto. Si el jurado considera que la alternativa propuesta por el demandante es razonable y elimina los riesgos del producto, éste será declarado defectuoso. El fabricante no podrá decir que su diseño no era defectuoso sólo porque otros fabricantes utilicen el mismo diseño. La industria entera puede ser culpable. El fabricante puede alegar la defensa conocida como el «state of art», según la cual cuando el producto fue construido el diseño era el «state of the art»; es decir, la ciencia no conocía un diseño alternativo más seguro. Con algunas excepciones, esta defensa es válida y protege al fabricante de una declaración de responsabilidad. 
to de información» ${ }^{28}$. Un fabricante de cigarrillos tiene la obligación de informar sobre los peligros para la salud que conlleva el consumo de sus productos, cuándo adquirió dicho conocimiento, o cuándo debía haber conocido que los cigarrillos eran peligrosos o potencialmente peligrosos. Los demandantes vieron frustradas sus demandas de reclamación de daños basadas en defecto de información porque la defensa alegaba con éxito que no había existido tal defecto de información. La defensa de las tabacaleras se basaba en la Federal Cigarette Labelling and Advertising Act de 1965. Esta Ley impone a los fabricantes la obligación de avisar a los consumidores de los riesgos asociados con el uso del tabaco ${ }^{29}$. En cumplimiento de la Federal Cigarette Labelling and Advertising Act las tabacaleras imprimieron en los paquetes de tabaco la frase: «Fumar puede ser peligroso para su salud». Aunque la Federal Labelling and Advertising Act fue un intento de informar a los consumidores sobre los riesgos de la utilización del tabaco, también sirvió para proteger a la industria del tabaco en contra de las acciones de responsabilidad. De hecho, los requisitos de etiquetaje federal han dado fuerza a la posición de la industria tabaquera en contra de las demandas de responsabilidad. Caso tras caso, la defensa argumenta que debido a que los consumidores habían sido notificados del peligro del consumo del tabaco, ellos mismos asumieron el riesgo asociado con el fumar, mientras que los demandantes alegan que la información contenida en los paquetes de cigarros era insuficiente e ineficaz como consecuencia de las prácticas publicitarias de las tabacaleras.

La Federal Labelling and Advertising Act también ha sido invocada como defensa de las tabacaleras para apoyar el razonamiento de que las demandas basadas en defecto de información están preempted. Los demandantes alegan que la información contenida en las cajetillas era in-

28 «Failure to warn». Un producto puede ser defectuoso si no está acompañado de la información necesaria para que su uso o consumo sea seguro. Sin embargo, si el producto podía haber sido diseñado con un mayor grado de seguridad, una información adecuada no convierte necesariamente un producto peligroso en seguro o en no defectuoso. Una información apropiada puede transformar ciertos productos peligrosos, que habrían sido defectuosos sin la información, en unos productos razonablemente seguros. Para que sea efectiva, la información debe ser completa, clara y debe informar al consumidor de la magnitud del riesgo.

29 Antes de 1985 el aviso era general y no tenía una información específica sobre las enfermedades relacionadas con su consumo. En octubre de 1985, una Ley federal obligó a los fabricantes a que incluyesen en el aviso una declaración de que podían desarrollar enfermedades del corazón, cáncer de pulmón y enfisema como resultado del consumo del producto. El aviso de 1996 es «Surgeon General's Warning: Quitting Smoking Now Greatly Reduces Serious Risk to Your Health». En los cigarros bajos en nicotina aparece «Warning: this product is not a safe alternative to cigarettes». 
suficiente y defectuosa; es decir, que existía información pero no era clara, concluyente o suficiente. El problema de esta argumentación no está sólo en que las tabacaleras cumplieron con el mandato de la Federal Labelling and Advertising Act al incorporar en las cajetillas la información en ella recogida, sino que la propia Ley en su sección 5 establece:

«No requirement or prohibition based on smoking and health shall be imposed under State law with respect to the advertising or promotion of any cigarettes the packages of which are [lawfully] labeled ${ }^{30}$.

La cuestión en disputa ${ }^{31}$ es la de si cabe un acción basada en defecto de información cuando la información incorporada al producto cumple los mandatos de una ley, y cuando además la propia ley impide que la información recogida sea otra. En otras palabras, si la acción está preempted por una ley federal, en este caso por la Federal Labelling and Advertising Act ${ }^{32}$.

${ }^{30}$ La versión de 1965 (que fue reformada por la Public Health Cigarette Smoking Act de 1969) era:

(a) No statement relating to smoking and health, other than the statement required by section 4 of this Act, shall be required on the cigarette package.

(b) No statement relating to smoking and health shall be required in the advertising of any cigarettes the

(c) packages of which are labeled in conformity with the provisions of this Act.

31 Todos los tribunales federales de apelación que han tratado la cuestión han resuelto a favor de que las demandas están preempted; por ejemplo, Kotler v. American Tobacco Co., 926 F.2d 1217, 1222-24 ( $1^{\text {st }}$ Cir. 1990), Pennington v. Vistron Corp., 876 F.2d 414, 420-421 ( $5^{\text {th }}$ Cir. 1989). Los tribunales estatales no han dado una respuesta tan favorables a la defensa de preempted; por ejemplo, Foster v. R.J. Reynolds Tobacco Co, 437 N.W.2d 655, 661-62 (Minn. 1989, Carlise v. Philp Morris Inc., 805 S.W.2d 498, 517 (Tex.Ct.App. 1991).

32 Un precedente judicial, anterior al caso Cipollone, se encuentra en el caso Dewey v. R.J. Reynolds Co., 121 N.J. 69, 577 A. 2d 1239 (1990). Wilfred Dewey falleció de cáncer de pulmón en 1980, después de fumar durante más de cuarenta años. Su mujer entabló una acción contra la compañía R.J. Reynolds alegando, entre otras causas de pedir, que existía un defecto de información sobre los riesgos del tabaco. El demandado contestó que la $\mathrm{Ci}$ garette Labelling Act impedía que prosperase dicha afirmación. El Trial Court declaró que la acción basada en defecto de información estaba preempted y el Tribunal de Apelación lo confirmó. Sin embargo, el Tribunal Supremo de New Jersey decidió que los tribunales de New Jersey podían declarar a un fabricante de tabaco responsable, a pesar de cumplir con el requisito federal (121 N.J. en 100, 577 A.2d en 1255). Esta decisión ha sido criticada por la doctrina (por ejemplo, ver NOTE, Tort Law-Strict Product Liability-New Jersey Supreme Court preserves Claims against Tobacco Companies. Dewey v. Reynolds Co., 121 MJ. 69, 577 A.2d 1239 (1990), Harvard Law Review, 1991, vol. 104, p. 1.723) porque consideran que la decisión tomada sólo se explica por la simpatía hacia las víctimas del ta- 
b. En concreto

En el caso Cipollone el jurado declaró:

1. que Liggett había incumplido su obligación de informar sobre los peligros del tabaco con anterioridad a 1966 (a partir de dicha fecha la acción esta preempted);

2. que el incumplimiento anterior fue la «causa próxima» de que Rose Cipollone fumara;

3. que el que Rose Cipollone fumase fue la causa próxima de su muerte.

(No se concedió indemnización de daños por defecto de información porque según la Ley de New Jersey de «comparative fault» no cabe indemnización si la culpa de la víctima supera el $50 \%$ de la culpa global. El jurado consideró que, debido a que de forma voluntaria había fumado, su responsabilidad en la causación de las lesiones era del $80 \%$ ).

4. Respecto a la demanda de incumplimiento de express warranty, el jurado encontró a Liggett responsable por incumplimiento de la garantía expresa hecha a los consumidores ${ }^{33}$, concedió a Antonio Cipollone la cantidad de 400.000 dólares como compensación por los daños sufridos por él, y no concedió indemnización a Rose Cipollone.

La relación entre las reglas de preemption y de comparative fault crearon una situación anómala que tuvo que ser tratada por el Tribunal de Apelación. El Tribunal de Distrito no distinguió entre la conducta de Rose Cipollone anterior a 1966 y posterior a 1965, cuando instruyó al jurado a considerar el grado de la culpa de Cipollone según el Derecho de New Jersey. Cipollone alegó que las instrucciones al jurado eran inconsistentes con el caso Ostrowski v. Azzara ${ }^{34}$, en el que el Tribunal

baco, y al intento de no impedir las posibles demandas futuras basadas en defecto de información. Se ha dicho también que, en este caso, el Tribunal sacrificó el razonamiento legal adecuado para mantener la puerta abierta en los tribunales de New Jersey de repartir los costes de una lesión particular causada por el tabaco entre los fabricantes y —como las indemnizaciones se traducen en un incremento de los precios del producto- entre la comunidad de fumadores.

33 N.J. Stat. Ann. 12a:2-313 (1) (a): «Any affirmation of fact or promise made by the seller to the buyer which relates to the goods and becomes part of the basis of fhe bargain creates and express warranty that the goods shall conform to the affirmation or promise.»

34111 N.J. 429, 545 A.2d 148 (1988). La señora Otrowski era una paciente cuya diabetes, una dieta pobre, y fumar causaron graves problemas de circulación. Como consecuencia tenía dolores en su pie izquierdo. El médico le recomendó la extirpación de la uña del 
Supremo de New Jersey declaró que una vez que ha ocurrido un ilícito legal, la conducta posterior de la víctima sólo debe ser considerada como forma de mitigar o reducir la indemnización. Lo que se pedía en la apelación era que no se aplicasen las reglas de la comparative fault sino las de avoidable consecuences. Esta última regla requiere que la víctima, siempre que pueda, ejercite un cuidado razonable para reducir los daños, y niega la parte de la indemnización que corresponde a los daños que deberían haber sido reducidos con un comportamiento adecuado de la propia víctima, pero que no lo fueron. La regla es diferente de la de comparative negligence, ya que la avoidable consecuences reduce la indemnización en proporción a la culpa de la víctima, pero no la impide. La regla reduce la indemnización por ítems concretos causados por la culpa de la víctima. La diferencia es importante, porque las reglas de avoidable consequences nunca operan como una defensa a la causa de acción de la víctima, sino sólo para eliminar ítems concretos de la indemnización.

El Tribunal de Apelación llegó a la conclusión de que la doctrina recogida en el caso Ostrowski debería ser aplicada también en Cipollone. Sin embargo, reconoció las dificultades que esta labor conlleva. Por un lado, no se puede analizar la conducta de Rose después de 1966 porque no cabe analizar la conducta de Liggett posterior a esa fecha, puesto que la acción está preempted. Por otro lado, el corte en el tiempo (antes de 1966 y posterior a 1966) es artificial, y los expertos se encontrarán con serias dificultades para establecer cuáles fueron los daños anteriores a 1966 y los posteriores a 1966, así como para saber cuál era el tanto por ciento de culpa de Rose en ambas fechas.

pie dolorido para permitir el drenaje. Ostrowski se sometió a la operación, y a pesar de que el médico le recomendó que dejase el tabaco, continuó fumando. Semanas después de la operación, se demostró que ésta no había sido eficaz y como resultado sufrió tres nuevas operaciones. En este caso, el jurado también encontró que la conducta del médico había sido negligente, pero consideró que la culpa de la víctima - basada en su comportamiento antes y después de la operación- era superior a la del médico, con lo que no reconoció que existiese derecho a la indemnización. El Tribunal de Apelación confirmó la decisión del Trial Court, pero el Tribunal Supremo dijo que la negligencia del médico se produjo en la operación y que la negligencia de la víctima era relevante en base a la comparative fault en el periodo de tiempo que transcurrió desde que comenzó el tratamiento hasta el momento de la operación, pero que, después de producida la operación, su actuar sólo podía ser considerado según las normas de la avoidable consequences. Se pidió al jurado que analizase la conducta de la víctima en estos dos momentos y si la negligencia de la víctima, durante el primer periodo de tiempo, era menor que la negligencia del médico en la operación. Si la respuesta era afirmativa existía derecho a la indemnización. La indemnización concedida debería ser reducida de acuerdo al comportamiento de la víctima en el segundo periodo de tiempo. 
El Tribunal Supremo afirmó que, en primer lugar, y respecto a la demanda por defecto de información, ésta estaba preempted desde 1966 (fecha de entrada en vigor de la Ley federal) y que por lo tanto no cabría interponer una demanda a partir de dicha fecha. En segundo lugar, la demanda por incumplimiento de express warranty basada en el Derecho de New Jersey ${ }^{35}$ no estaba preempted. El Tribunal reconoció que las pruebas de la garantía contraída por el fabricante se basaban principalmente en la publicidad y en la información del producto, pero que la obligación la había contraído el fabricante y no era una obligación impuesta por el Derecho estatal ${ }^{36}$. En tercer lugar, el Tribunal Supremo dijo que la demanda basada en las declaraciones falsas (fraudulenf misrepresentation), realizadas en la publicidad de los demandados no estaba preempted ${ }^{37}$. En cuarto lugar, respecto a la demanda de conspiración para falsear u ocultar hechos materiales concernientes a los peligros del tabaco para la salud, contestó que tampoco estaba preempted ${ }^{38}$.

Se pensó que el caso Cipollone resolvía los problemas ocasionados en el pasado con la defensa de preempted. Sin embargo, un sector de la doctrina opina que esta decisión ha creado aún más confusión ${ }^{39}$, y que sus contradicciones internas y la falsa distinción llevada a cabo en la sentencia hacen que su aplicación en los tribunales resulte extremadamente difícil.

35 12A:2-313 (1) (a) N.J.Stat.Ann: «Any affirmation of facts or promise made by the seller to the buyer which relates to the goods and becomes part of the basis of the bargain creates an express warranty that the goods shall conform to the affirmation or promise». 12A:2-313 (1) (a) N.J.Stat.Ann.

36 El Tribunal dijo:

"That the terms of the warranty may have been set forth in the advertisements rather than in a separate documents is irrelevant to the preemption issue (though possibly not to the state law issue of whether the alleged warranty is valid and enforceable) because although the breach of warranty claim is made "with respect to advertising" it does not rest on a duty imposed under state law. Accordingly, to the extent that petitioner has a viable claim for breach of express warranties made by respondents, that claim is not pre-empted by the 1969 Act.»

37 «Thus, we conclude that the phrase "based on smoking and health" fairly but narrowly construed does not encompass the more general duty not to make fraudulent statements. Acoordingly, petitioner's claim based on allegedly fraudulent statements made in respondents'advertisements are not pre-empted by 5(b) of the 1969 Act.»

38 "The predicate duty underlying this claim is a duty not to conspire to commit fraud. For the reasons stated in our analysis of petitioner's intentional fraud claim, this duty is not pre-empted by 5(b) for it is not a prohibition "based on smoking and health" as that phrase is properly construed.»

39 Ver, NOTE, Federal Preemption of State Law, Harvard Law Review, 1992, vol. 106, pp. 347 y ss. 


\section{Después de Cipollone}

El año 1992 parece ser la fecha de inicio de lo que se ha dado en conocer como el tercer movimiento en la «guerra contra el tabaco». El caso Cipollone no fue importante sólo por aclarar, en mayor o menor medida, la defensa de preempted, sino también porque en él se presentaron, por primera vez, los que luego serían conocidos como los «documentos Brown and Williamson». Estos documentos aparecen, en su totalidad, el 12 de mayo de 1994 en la Universidad de California ${ }^{40}$. Los documentos suponen unas 4.000 páginas compuestas de informes, memorándums y cartas generadas por la corporación Brown and Williamson y la British American Tobacco Company durante un periodo de más de 30 años. Se publicaron en $1995^{41}$. De ellos se desprende que las investigaciones conducidas por las tabacaleras sobre los efectos negativos del tabaco en la salud eran más sofisticadas y avanzadas que los estudios de la comunidad médica. También, que existieron debates entre los directivos de las tabacaleras, una vez conocidos los efectos del tabaco y la naturaleza adictiva de la nicotina, para decidir si se hacían públicos y la decisión tomada fue la de no hacerlos públicos; y que la industria del tabaco adoptó una estrategia uniforme que consistía en negar la relación entre las enfermedades y el consumo de tabaco, y el que la nicotina fuese adictiva.

La aparición de los documentos «Brown and Williamson» provocó la interposición de cientos de demandas, tanto de demandantes individuales, como de los Attorneys General ${ }^{42}$ de los estados. Así mismo, motivó la aparición de las demandas de la industria tabaquera contra la admisión en juicio como prueba de dichos documentos. Estos documentos también han tenido como resultado que a las tabacaleras se les

40 En un primer momento se pensó que habían sido enviados por una fuente anónima, más tarde se supo que la persona que los había enviado era un antiguo empleado de Brown and Williamson que los fotocopió durante su empleo.

41 Journal of American Medical Association, 19 de julio de 1995.

42 Black, H.C., Black's Law Dictionary, St.Paul, Minn., West Publishing Co., 1991, p. 86:

"Attorney General: The Attorney General, as head of the Department of Justice and chief law officer of the Federal Government, represents the United States in legal matters generally and gives advice and opinions to the President and to the heads of the executive departments of the Government when so requested. The Attorney General appears in person to represent the Government in the U.S. Supreme Court in cases of exceptional gravity or importance.

In each state there is also an attorney general, who is the chief law officer of the state. He gives advice and opinions to the governor ant to executive and administrative departments and agencies.» 
demande, no sólo por las teorías anteriores de responsabilidad por productos defectuosos, sino que cobren mayor importancia las alegaciones de conspiración, fraude, falsedad, etc.

Una de las demandas que más repercusión ha tenido en los medios de comunicación ha sido la entablada por la viuda del «hombre Malboro» en su nombre y en el de su marido, Lilo McLean, el 30 de agosto de 1996. En esta demanda se abandonan las alegaciones pasadas de defecto de diseño y de información para centrar la cuestión en el fraude, el incumplimiento de express warranty e implied warranty, la negligent misrepresentation y la misrepresentation to consumers. Además, los demandados no son únicamente las tabacaleras productoras del tabaco que fumaba la víctima, sino todas las tabacaleras, el Council for Tobacco Research y el Tobacco Institute Inc. Se alega que la responsabilidad de los demandados surge del hecho de que todos tomaron parte en una conspiración que dio lugar a la comisión de todos o de algunos de los actos ilícitos alegados anteriormente, y porque intencional y conscientemente causaron lesiones o enfermedades.

Otra peculiaridad de esta demanda es que uno de los daños que se alegan es el de que David McLean se hizo adicto a la nicotina. Se abandonan las lesiones, la enfermedad o la muerte como daños, y se recorre a la adicción a la nicotina ${ }^{43}$.

Otro cambio que se ha producido ha sido el de modificar el producto que se considera defectuoso. Se ha pasado del cigarrillo a la boqui1la. Un ejemplo es un caso en San Francisco en el que se indemnizó a una persona de 72 años, fumadora de cigarillos Kent, con dos millones de dólares (1.300.000 como daños compensatorios y 700.000 como daños punitivos). La demanda se interpuso contra el fabricante del tabaco (Lorillard) y contra el suministrador del filtro (Hollingsworth \& Vose). La cuestión en el juicio era la de si la mesotelioma que sufría el demandante fue causada por la utilización de asbesto en los filtros desde 1952 a 1956. El demandante había dejado de fumar en 1963. Los demandados apelaron y perdieron ${ }^{44}$.

En suma, los fracasos continuos de los fumadores en sus demandas por defecto de información y de diseño, junto con la decisión de Cipollone, han llevado a sus abogados a plantear nuevas estrategias, como la contemplada en el caso McLean (Malboro man): modificar las cau-

43 Sobre esta demanda todavía no ha existido sentencia firme.

44 De no llegar a un acuerdo entre las partes, el fabricante del cigarro deberá abonar 1.200.000 dólares y el fabricante del filtro 790.000 dólares Información recogida en Product Liability International, septiembre, 1995, p. 137, y en Liability Risk and Insurance, febrero de 1998, p. 11. 
sas de pedir, los demandados, y el daño. El éxito de estas nuevas demandas todavía está por ver, ya que la gran mayoría, o están en el District Court, o en apelación.

\section{Fumadores pasivos}

El consumo de tabaco no sólo tiene efectos nocivos entre aquellos que lo consumen de forma directa sino, también, para aquellos que sin ser fumadores respiran el humo de los cigarros de forma indirecta; los «fumadores pasivos». El humo del tabaco ha planteado una nueva cuestión: ¿son los empleadores responsables de las enfermedades pulmonares contraídas por sus empleados en los centros laborales, si se demuestra que éstas han sido contraídas por la exposición al humo de tabaco?

En el Reino Unido, la señora Ashby, de 49 años de edad, recibió 2.500 libras de su empleador (el London Bank) en un acuerdo extrajudicial celebrado en 1995. La mujer trabajaba en lo que se consideraba un área libre de tabaco. No obstante, los fumadores frecuentaban la zona. La Sra. Ashby ingresó en el hospital en 1991, en donde se le diagnosticó asma, y posteriormente fue declarada incapaz laboral ${ }^{45}$.

El punto de partida es que los empleadores son responsables por no haber prohibido el consumo de tabaco en el centro de trabajo, puesto que supone un riesgo para la salud. Las demandas de los fumadores pasivos contra sus empleadores han vuelto a plantear antiguos problemas, especialmente el de la prueba de la relación de causalidad. La comunidad médica ha publicado informes en los que se estudia la relación entre la inhalación del humo de tabaco por los fumadores pasivos y las

45 Product Liability International, febrero 1995, pp. 23. Otro caso del Reino Unido que terminó en acuerdo extrajudicial fue el de Verónica Bland. Bland demandó a su empleador, el Stockport Council, alegando que su bronquitis crónica había sido causada por la exposición al humo de los cigarrillos de sus compañeros de trabajo. Recibió 15.000 libras. En Gran Bretaña, la sección 2(1) de la Health and Safety at Work Act imponía al empleador la obligación general «to ensure, so far as reasonably practicable, the health, safety and welfare at work of all his employees». El comportamiento del empleador se valorará de acuerdo con el criterio de «un empleador razonable y prudente», en base a lo que conocía o debía haber conocido. En su actuación el empleador debe «sopesar los riesgos, en términos de la posibilidad de que ocurra la lesión y las consecuencias potenciales de ésta si ocurre; debe comparar el resultado anterior con la efectividad de las precauciones a tomar y los gastos e inconvenientes que estas suponen». Stokes v. Guest Keen \& Nettlefold (Bolts \& Nuts) Limited (1968) 1 WLR 1776, p. 1.783) recogido en Environmental Tobacco Smoke, loc. cit., p. 9. Vid., Workplace (Health, Safety and Weltare) Regulations 1992, en vigor desde enero de 1993 para establecimientos laborales nuevos y desde enero de 1996 para los ya existentes. 
enfermedades pulmonares, pero sus datos son difíciles de interpretar y su importancia difícil de valorar. Un estudio del Reino Unido, de 1988, Fourth Report of the Independent Scientific Committee on Smoking and Health (conocido como el «Froggart Report») concluyó que las pruebas epideomológicas realizadas apoyaban la existencia de un «pequeño aumento» del riesgo de cáncer de pulmón (el aumento del riesgo sería del 10 al 30\% entre los no fumadores expuestos al humo del tabaco) ${ }^{46}$. En los Estados Unidos la Environmental Protection Agency (EPA), en 1993, clasificó el humo medioambiental como un grupo cancerígeno, pero no lo consideró como una de las causas de las enfermedades respiratorias de los adultos.

Estas demandas tienen a su favor el hecho de que muchas de las defensas que se plantearon con éxito frente a los fumadores activos no tienen cabida en este supuesto. Pensemos, por ejemplo, en la «asunción de riesgo». No cabe alegar que el fumador pasivo asumió el riesgo de fumar, ya que no fumó, y además no pudo evitarlo al encontrarse en su centro de trabajo. Tampoco cabe la defensa de compensación de culpas, ya que en ningún momento se puede apreciar comportamiento negligente en la conducta de la víctima; se limitó a trabajar y a respirar. Sin embargo, su éxito no ha sido el que se esperaba.

El principal problema al que se enfrenta el trabajador (fumador pasivo) al entablar estas acciones es que, aunque demuestre que el humo medioambiental es una causa posible de su lesión, ésta será sólo una causa posible dentro de una serie de causas posibles. La carga de la prueba de que fue la exposición al humo medioambiental la causa de sus lesiones, y no cualquier otra causa, la tiene el trabajador. Debido a que el trabajador ha estado expuesto al humo del tabaco en otras facetas de su vida, como por ejemplo en su propio hogar o en las relaciones con sus amigos, la prueba es extremadamente difícil o imposible ${ }^{47}$, por lo que parece que el éxito de estas demandas sea, como mucho, reducido ${ }^{48}$.

46 Este informe explica que un incremento de este tipo «...significa que la gente que nunca ha fumado, pero que ha sido expuesta a ETS la mayor parte de sus vidas, tienen un riesgo de $10-30 \%$ de tener cáncer de pulmón frente a los que no han sufrido esta exposición... así que podría haber de 1 a 3 pacientes extras de cáncer de pulmón al año de cada 100.000 no fumadores expuestos al humo medioambiental». Ver Meltzer, J., Environmental Tobacco Smoke. A realistic perspective on smoking and the workplace, Product Liability International, enero, 1994, pp. 8 y ss.

47 La class action entablada por los asistentes de vuelo en los Estados Unidos presenta peculiaridades que serán tratadas más adelante.

${ }^{48}$ En Australia, el Departamento de Salud de Western Australia demandó al casino Burswood por fracasar en imponer las medidas efectivas que controlasen el humo ambiental, de tal forma que la salud y la seguridad de sus empleados no sufriese riesgos. La de- 
El leading case, en los Estados Unidos, es Palmer v. Del Webb's High Sierra ${ }^{49}$. Palmer, no fumador, trabajó en el Casino durante más de 20 años. Su trabajo consistía en supervisar las mesas de juego dentro del Casino, donde había altos niveles de humo de tabaco (la empresa regalaba cigarrillos a los jugadores). A la edad de 58 años, Palmer contrajo una bronquitis y asma ${ }^{50}$. Palmer no consiguió que su enfermedad se catalogase como enfermedad laboral y con ello lograr la indemnización, porque la Ley NRS 617.440 requiere que la enfermedad laboral sea un incidente del empleo y no meramente una consecuencia accidental que no está relacionada con la naturaleza del trabajo. La sección 617.440(1) específicamente establece que la enfermedad debe ser «a natural incident of the work as a result of the exposure occasioned by the nature of the employment». El Tribunal Supremo de Nevada dijo:

"What this language means is that the disease must arise out of job conditions, especifically, the "nature of the employment". [T]here must be a connection between the kind of job and the kind of disease. Mere causation is not enough. One could easily say that going to work caused a person to develop ulcers; but the "nature of the employment" is, in most cases, not inherently ulcerogenic; and ulcers are not in all probability a "natural incident of the work" calimed to be the cause of the disease. Thus, a person who develops ulcers, catches a cold or gets a migraine headache on the job is probably not going to be able to assert a successful claim for occupational disease compensation.»

El Tribunal no consideró que el humo del tabaco, a pesar de presentarse comúnmente en bares y casinos, sea incidental al carácter de dichos negocios; es decir, que no es una incidencia natural de la actividad laboral, como podría ser el respirar polvo de carbón para un minero. Además, el Tribunal manifestó que es labor del Parlamento de Nevada la de incluir a los fumadores pasivos trabajadores de bares y casinos como grupo legitimado para acceder a una compensación por enfermedad laboral, pero que ellos no tienen dicho poder. Añadió que no se considera probada la relación de causalidad porque la exposición al humo de tabaco se da también fuera de los lugares de trabajo.

manda fracasó por falta de pruebas. El magistrado concluyó que, dado el conflicto de opiniones entre los expertos, el Tribunal no podía decidir sobre cuál era la opinión correcta. Vid., Environmental Tobacco Smoke, loc.cit., p. 9

49 Supreme Court of Nevada, 1992. 108 Nev. 673, 838 P.2d 435.

${ }^{50}$ La demanda se entabla para lograr que se catalogue la enfermedad de Palmer como enfermedad laboral cubierta por la Occupational Disease Act de Nevada. 


\section{II. «Class Action» v. Tabacaleras}

La estrategia contra las tabacaleras ha adquirido, en la última década, la forma de la class action, figura desconocida en nuestro Derecho ${ }^{51}$. El Black's Law Dictionary la define como:

«A "class" within rule relating to class action must be taken in broad colloquial sense of group of people ranked together as having common characteristics, and the function of the enumerated requirements of rule is to assure that from those characteristics there arises a common legal position vis-à-vis the opposing party, the legal right or obligations of which the court can efficiently and fairly adjudicate in a single proceeding ${ }^{52}$.

La interposición de una class action permite que uno o más demandantes interpongan una acción en nombre de todo un grupo, sin necesidad de intervención directa de los miembros del grupo, sino sólo la de los representantes ${ }^{53}$. Tal vez, lo más sorprendente de este tipo de accio-

51 Ver De Angel YÁgüEz, R., Algunas previsiones sobre el futuro de la responsabilidad civil (con especial atención a la reparación del daño), Madrid, Civitas, 1995, p. 94:

«La legislación procesal no contempla supuestos de "legitimación de grupo", que atribuyan a una persona "representación" para actuar en nombre de todos los demás afectados por el mismo motivo aunque éstos no litiguen.

No parece que la alusión del artículo 9.2 de la Constitución a los "grupos en que se integra" el individuo sea bastante para dar carta de naturaleza entre nosotros a figuras como la class action. Está por ver qué aplicación pueden hacer los órganos jurisdiccionales del acaso más expresivo artículo 7 de la Ley Orgánica del Poder Judicial de 1 de julio de 1985, ...»

52 Black, H.C., Black's Law Dictionary, 6. a edic., St. Paul, Minn., West Publishing Co., 1991, p. 170. Vid. Landers, J.M., MartÍn, J.A., Yeazell, S.C., Civil Procedure, 2. edic., Boston, Little Brown and Co., 1988, p. 546:

"Although one can trace litigation by groups into the mists of the common law, the modern class action traces its inmediate pedigree only to the seventeenth century. In a series of cases involving rural tenants and parishioners suing their landlords and persons over customary duties, Chancery began to develop me doctrines that would permit a few active litigants to represent the rest.»

Sobre la historia y evolución de la class action, YEAzell, S.C., Group Litigation and Social Context: Toward a History of the Class action, Columbia Law Review, 1977, vol. 77, p. 866.

53 Aceptada la class action por el Tribunal, éste lo pondrá en conocimiento de sus miembros mediante una notificación individual para que puedan, expresamente, separarse del resultado futuro de la demanda o participar de su resultado. Los requisitos de la notificación se recogen en la Rule 23 (c)(a) Fed. R. Civ. P. La notificación a los miembros del grupo es una labor difícil y no exenta de problemas, como se puede ver, por ejemplo, en Eisen v. Carlisle \& Jacquelin (417 U.S. 156 (1974)) y Phillips Petroleum v. Shutts (472 U.S. 797 (1985)). 
nes para un jurista continental sea que, si se admite la class action, los efectos de la sentencia se trasladarán a todos los miembros del grupo . De la class action se ha dicho que es «uno de los remedios socialmente más útiles de la historia» ${ }^{54}$, pero también que supone «un chantaje legal» ${ }^{55}$. Las opiniones dispares no se corresponden siempre con la división: demandantes, demandados. Aunque los demandados usualmente tienen mucho que ganar en una class action, algunos consideran que se beneficiarán defendiendo cada demanda de forma separada, en la creencia de que lograrán negociaciones más baratas o que desincentivarán las demandas individuales. Los abogados de los demandantes, normalmente, se niegan a la class action porque aquellos que han sufrido daños graves esperan lograr una mayor indemnización si actúan de forma independiente. Por el contrario, los que han sufrido daños menores apoyan la class action ya que esperan obtener un beneficio del poder negociador del grupo ${ }^{56}$.

Las class actions entabladas contra las tabacaleras están muy influenciadas, en sus planteamientos, por la decisión del caso Cipollone y por la aparición de los documentos «Brown and Willianson».

Las principales class actions son:

1. Acción colectiva entablada por los fumadores adictos a la nicotina (conocida como el caso Castano).

2. Acción colectiva entablada por los asistentes de vuelo expuestos al «humo del tabaco en el ambiente de los aviones» (conocida como el caso Broin).

3. Acción colectiva entablada por los consumidores de productos de tabaco bajo en nicotina.

4. Acción colectiva entablada por los fumadores residentes en el estado de Florida.

El primer escollo al que se presentan los demandantes que buscan constituir una class action es el de lograr una certification order; es decir, que los tribunales les reconozcan como representantes de un grupo con legitimación para interponer la demanda. Para ello debe cumplir con los prerequisitos contenidos en la regla 23 (a) de la Federal Rules of Civil Procedure. La regla 23 (a) recoge que uno, o más, de los

54 Pomerantz, New Developments in Class Actions-Has Their Death Knell Been Sounded?, Bussines Law, 1970, vol. 25, pp. 1.259 y ss.

55 HANDLER, The Shift from Substantive to Procedural Innovations in Anti-Trust Suits, Columbia Law Review, 1971, vol. 71, pp. 1 y ss.

56 Ver Fleming, J.G., The American Tort Process, Oxford, Clarendon Press, 1988, pp. 244 y ss. 
miembros de un grupo puede demandar o ser demandado como representante del mismo, sólo si:

(1) el grupo es tan numeroso que la unión de todos sus miembros es impracticable,

(2) existen cuestiones de hecho y de derecho comunes a todo el grupo,

(3) las demandas o las defensas de los representantes son comunes a las demandas o defensas del grupo,

(4) los representantes protegerán de forma justa y adecuada los intereses del grupo.

Si el tribunal considera que se cumplen los prerequisitos anteriores pasará a analizar la cuestión bajo la regla 23 (b) Federal Rules of Civil Procedure, que es la que más problemas presenta en la búsqueda de la certification order, especialmente la sección 23 (b) 3, en la que se recogen los requisitos de «superior» $\mathrm{y}$ «predominate ${ }^{57}$.

57 Rule 23(b) Class Actions Maintainable.

An action may be maintained as a class action if the prerequisites of subdivision (a) are satisfied, and in addition:

(1) the prosecution of separate actions by or against individual members of the class would create a risk of

(a) inconsistent or varying adjudications with respect to individual members of the class which would establish incompatible standards of conduct for the party opposing the class, or

(b) adjudications with respect to individual members of the class which would as a practical matter be dispositive of the interests of the other members not parties to the adjudications or substantially impair or impede their ability to protect their interests; or

(2) the party opposing the class has acted or refused to act on grounds generally applicable to the class, thereby making appropriate final injunctive relief or corresponding declaratory relief with respect to the class as a whole: or

(3) the court finds that the questions of law or fact common to the members of the class predominate over any questions affecting only individual members, and that a class action is superior to other available methods for the fair and efficient adjudication of the controversy. The matters pertinent to the findings include: $(A)$ the interest of members of the class in individually controlling the prosecution or defense of separate actions; $(B)$ the extent and nature of any litigation concerning the controversy already commenced by or against members of the class; $(C)$ the desirability or undesirability of concentrating the litigation of the claims in the particular forum; (D) the difficulties likely to be encountered in the management of a class action.

Un buen ejemplo de casos en los que se ha negado la certification order en base a la regla 23 (b) lo constituyen los relacionados con el producto Dalkon Shield, ver por ejemplo, 693 F.2d 847 ( $9^{\text {th }}$ Cir. 1982). 


\section{Castano v. Ameritan Tobacco Co.}

En 1995 se entabló la que, de haberse admitido, habría sido la demanda colectiva más grande jamás planteada ante la jurisdicción federal; nos referimos al caso Castano v. Ameritan Tobacco Co ${ }^{58}$. El Tribunal definió al grupo demandante como:

a) todas las personas dependientes de la nicotina, residentes en los Estados Unidos (...) que hayan adquirido o fumado cigarros fabricados por los demandados, a partir de 1943;

b) los administradores, representantes y las herencias de los fumadores adictos a la nicotina, a partir de 1943;

c) los cónyuges, hijos y «otros relevantes» de los fumadores adictos a la nicotina, a partir de 1943.

La demanda se basaba en la alegación de que los demandados fracasaron fraudulentamente en informar a los consumidores que la nicotina es adictiva y manipularon el nivel de nicotina de los cigarros con el fin de mantener su naturaleza adictiva. La class action alega 9 causas de pedir: fraud and deceit, negligent misrepresentation, intentional infliction of emotional distres, negligence and negligent infliction of emotional distress, violation of state consumer protection statutes, breach of express warranty, breach of implied warranty, strict product liability y redhibition en base al Código civil de Louisiana ${ }^{59}$. Uno de los argu-

58160 F.R.D. 544, 560 (E.D. La.1995).

${ }^{59}$ Los demandantes propusieron un juicio con un plan de 4 fases. En la fase 1, el jurado deberá determinar cuestiones comunes como el nivel de responsabilidad. Esta fase incluirá cuestiones como (1) cuestiones de derecho y de hecho en relación a la conducta de los demandados, su responsabilidad negligente; (2) cuestiones de hecho y de derecho sobre la alegación de conspiración y de acción concertada de los demandados; (3) cuestiones de hecho relacionadas con la naturaleza adictiva, la dependencia, las características y propiedades de la nicotina; (4) cuestiones de hecho sobre la nicotina de los cigarros como productos defectuosos; (5) cuestiones de hecho sobre si la conducta ilícita de los demandados fue intencional, descuidada o negligente; (6) identificación de cuáles de los demandados enfocaron sus esfuerzos promocionales y su publicidad hacia grupos particulares (jóvenes, minorías); (7) availability of a presumption of reliance; (8) sobre si la supresión, por parte de los demandados, de hechos sobre la naturaleza adictiva de la nicotina niega la posibilidad de utilizar como defensa la de «personal choice»; (9) la responsabilidad de los demandados por los daños actuales y las categorías de dichos daños; (10) la responsabilidad de los demandados por emotional distress; y (11) la responsabilidad de los demandados por daños punitivos.

En la segunda fase el jurado debería determinar la indemnización compensatoria, y debería establecer un ratio basado en la indemnización de los daños compensatorios para establecer el quantum de los daños punitivos.

La tercera fase consistiría en un procedimiento complicado para determinar la indemnización compensatoria de cada miembro individual de la class. 
mentos principales de los demandantes, en orden a lograr la certificación del grupo, era el de que la forma de class action es superior a los procesos entablados por los particulares, porque con ella se evitaría la saturación de los tribunales con miles de demandas idénticas.

El caso Castano constituye un buen ejemplo de la aplicación de la regla 23 (b) para negar la certification orden del grupo, y supuso el fracaso de la acción colectiva que pretendía terminar con la «guerra del tabaco» (inicialmente, el grupo fue definido como «all nicotine dependene persons in the United States»).

El Tribunal de Distrito acordó la certificación de la class action, en parte, concluyendo que se habían cumplido los requisitos de la Federal Rule 23 (a), pero en la apelación el resultado fue contrario a la certificación. El Tribunal de Apelación consideró que la decisión del Tribunal de distrito era errónea, y que no se podía conceder la certificación de la acción colectiva porque no se cumplían los requisitos de la Federal Rule (23)(b)(3) (donde se recoge que la acción colectiva puede entablarse cuando el tribunal considere que las cuestiones de hecho y de derecho comunes a los miembros del colectivo son superiores a aquellas cuestiones que sólo afectan a miembros individuales, y que la acción colectiva es superior a los otros métodos disponibles para una resolución justa y eficiente de la controversia). Uno de los factores que más peso tuvieron en la decisión del Tribunal de Apelación fue la consideración de los graves problemas de administración que se plantearían en esta acción; como, por ejemplo, la determinación del Derecho aplicable, las notificaciones a millones de miembros del colectivo, las dificultades de los procedimientos para determinar quiénes son los adictos a la nicotina, etc. Por éstas y otras razones el Tribunal de Apelación negó la certificación de la class action ${ }^{60}$.

El Tribunal de Apelación consideró que el Tribunal de Distrito erró en su análisis del Derecho aplicable. El requisito de que el tribunal conozca el Derecho que se aplicará al grupo es especialmente importante cuando existen diferencias entre los Derechos de los estados. En este caso, el Tribunal de Distrito no tuvo en cuenta de forma adecuada cómo afectarían las variaciones en el Derecho estatal ${ }^{61}$ del requisito de

En la cuarta fase el tribunal aplicaría el ratio establecido para el cálculo de los daños punitivos y podría dar lugar a una revisión de la indemnización.

60 Posteriormente, los mismos abogados que entablaron la acción colectiva de Castano han entablado otras acciones colectivas, pero ahora, en el nivel estatal, la gran mayoría todavía están pendientes de resolución.

${ }^{61}$ Por ejemplo, algunos estados no reconocen la strict liability (Cline v. Prowles Indus., 418 A.2d 968, 979.980 8Del. 1980). Aunque la mayoría ha adoptado el Restatement (Second) of Torts existen diferencias entre ellos, ver SPEISER, S.M., KRAUSE, G.H., Guns, 
«predominance». A pesar de que el Tribunal de Distrito reconoció que existirían importantes problemas a la hora de manejar el proceso, llegó a la conclusión de que dichos problemas eran de menor peso que «la posibilidad de miles - si no de millones — de juicios similares en miles de juzgados de toda la nación».

Además, se tuvo en cuenta que permitir una class action de estas características crearía una gran presión sobre los demandados para que negociasen, presión mucho mayor que la ejercida por demandas individuales. El riesgo de enfrentarse a un veredicto de todo o nada supone un riesgo demasiado alto, aún cuando la probabilidad de un veredicto adverso sea pequeña. Por eso no sorprende que sólo unos pocos supuestos logren una orden de certificación de la class action en los tribunales. En la mente del Tribunal de Distrito estaba el recuerdo de los juicios y de las class action anteriores planteadas en los casos de asbestos $^{62}$. Estos casos enfrentaron al sistema judicial norteamericano a miles de procesos en todo el país, y en aquel tiempo se habló de una posible crisis del poder judicial. El Tribunal de Apelación no aceptó que ambas situaciones fuesen comparables. En su opinión, hay una diferencia fundamental entre ambos supuestos: en las demandas contra las tabacaleras, la posibilidad de que una crisis del poder judicial se materialice puede no ser cierta. El Tribunal dijo:

«The plaintiffs'claims are based on a new theory of liability and the existence of new evidence. Until plaintiffs decide to file individual claims, a court cannot, from the existence of injury, presume that all or even any plaintiffs will pursue legal remedies ${ }^{63}$.

\footnotetext{
A.W., The Ameritan Law of Torts, New York, The Lawyers Cooperative Publishing co., 1986, cap. 18. Otras importantes diferencias las encontramos en la defensa de asunción de riesgo. En algunos estados funciona como defensa absoluta (S.C. Code Ann. 15-73-20 (Law Co-op 1976)), mientras que en otros funciona como parte del análisis de la compensación de culpas (Colo.Rev.Stat. 13-21-111.7 (1986)).

62 En el caso conocido como School Asbestos (789 F.2d, 1010) se certificó la class action a pesar de existir variaciones entre los derechos estatales, pero el Tribunal dijo:

«To meet the problem of diversity in applicable state law, class plaintiffs have undertaken an extensive analysis of the variances in products liability among the jurisdictions. That review separates the law into four categories. Even assuming additional permutations and combinations, plaintiffs have made a creditable showing, which apparently satisfied the district court, that class certification does not present insuperable obstacles. Although we have some doubt on this score, the effort may nonetheless prove successful.»
}

${ }^{63}$ En su opinión, el tribunal no puede extrapolar del número de demandantes potenciales el número real de casos que se entablarán en los tribunales. Según estudios sólo del 10 al $20 \%$ de las personas que sufren un daño recurren a los tribunales. 
También llevó a cabo una interesante distinción entre mass torts «inmaduros»y «maduros». Los mass torts «inmaduros» —aquellos sobre los que se han emitido pocos veredictos- deben ser analizados entre demandantes individuales, hasta que las cuestiones de relación de causalidad, daños típicos y niveles de indemnizaciones concedidas se haya establecido. A partir de este momento se convierte en un mass tort «maduro» - como en su día lo fueron los casos de asbesto o del Dalkon Shield - y entonces se puede recurrir a procedimientos como la class action, no antes.

La alegación final de los demandantes fue que los juicios individuales no son adecuados porque el tiempo corre en su contra. Este argumento fracasa porque, en primer lugar, no es cierto que el tiempo corra en su contra - en el sentido de que muchos de los posibles integrantes del grupo fallecerán y no podrán pertenecer a él- ya que cabe la posibilidad de integrarse en el grupo a sus familiares o a la herencia. En segundo lugar, los pleitos entablados en décadas anteriores no sirven como referencia porque las causas de pedir y las pruebas practicadas son diferentes de las resueltas en el pasado.

Por todo ello, el Tribunal de Apelación negó la orden de certificación de la class action. Idéntico resultado se alcanzó en Tribunal Supremo ${ }^{64}$.

\section{Broin v. Philip Morris ${ }^{65}$}

El 22 de noviembre de 1994 se planteó la que sería la primera class action que conseguiría una orden de certificación firme; Broin v. Philip Morris $^{66}$.

64 Castano v. American Tobacco Co, 84 F.3d 734 (5 $5^{\text {th }}$ Cir. 1996). Un año después de la decisión del Tribunal Supremo, el 22 de mayo de 1997, los abogados de los demandantes han planteado una nueva class action ante un tribunal federal con idénticos presupuestos a favor de los fumadores de 35 estados y del Distrito de Columbia. Todavía no resuelta. También han intentado lograr una orden de certificación para un grupo que no sería el de todos los afectados por la nicotina en los Estados Unidos, tan sólo en estados concretos; por ejemplo, Steven Arch v. The Ameritan Tobacco Company, Inc, planteada en agosto de 1996 en la Court Of Common Pleas of Philadelphia County, Pa., de esta forma tratan de evitar el problema de la ley aplicable. En mayo de 1997 se negó la orden de certificación en un supuesto idéntico por un tribunal de Kansas City, Missouri.

65 Información sobre este caso se encuentra en INTERNET, http://www.Kinsella. com/broin/

${ }^{66}$ El Tribunal previamente consideró este caso, el 20 de abril de 1992, y negó la orden de certificación del grupo, la cual fue apelada ante el Third District Court of Appeal y anulada por éste (Broin v. Philip Morris Companies, Inc., 641 So.2d 888 (Fla.1994)). El Tribunal de Apelación consideró que la orden de certificación era apropiada en este supuesto y remitió de nuevo el caso al Tribunal de Distrito. 
Los demandantes/representantes del grupo son 25, y representan a unos 60.000 asistentes de vuelo. El grupo de los demandantes es el de todos los asistentes de vuelo no fumadores que han sido o están empleados por líneas aéreas con base en los Estados Unidos, y que sufren de enfermedades o desórdenes causados por su exposición al humo de los cigarros que se fuma en los aviones. Estamos ante un supuesto de lesiones a fumadores pasivos ${ }^{67}$. Piden indemnización basándose en las teorías de responsabilidad objetiva, incumplimiento de garantía implícita, negligencia, fraude, engaño y conspiración para cometer fraude. Los demandados son todas las tabacaleras de los Estados Unidos y otras asociaciones relacionadas con ellas.

Se alega que la acción de los demandantes es idéntica: la inhalación, de forma pasiva, del humo provocado por el consumo de tabaco. También los demandados actuaron contra los asistentes de vuelo de la misma manera: fabricando los cigarrillos de los que se desprendía el humo. Además, todos piden indemnización bajo los mismos intereses y comparten el interés común de obtener compensación por sus lesiones. El Tribunal consideró que los demandados planteaban defensas idénticas contra estas demandas. El Tribunal también advirtió que el tratamiento como grupo ayudará a la eficacia y a la economía judicial —evitando la duplicidad de procesos sobre cuestiones comunes- y que el hecho de que las lesiones de los demandantes no sean idénticas no impide el reconocimiento del grupo. Además, apreció el hecho de que los demandados tienen grandes recursos económicos para afrontar la defensa de demandas individuales, pero los demandantes carecen de dichos recursos.

En el caso Broin se aplicó la Rule 1.220(a)(2) del Florida Rules of Civil Procedure ${ }^{68}$ y se declaró que se cumplían los cuatro prerequisitos exigidos para conceder una certification order (numerosity, commonality, tipicality y adecuacy of representation). Respecto al requisito de la Rule $1220(\mathrm{~b})(3)^{69}$, de que la class action sea un método superior a los

67 Broin v. Philip Morris, No. 91-49738 CA (Fla.Cir., 11 th Jud. Dist., Dade County).

Aunque la Environmental Protection Agency ha declarado el humo de los cigarros como cancerígeno, en la misma categoría que el asbestos, las tabacaleras siguen cuestionando que el humo sea cancerígeno. Un artículo del Journal of Ameritan Medical Association estableció que la industria «de forma privada había conducido investigaciones internas; al menos algunas de ellas apoyan la conclusión de que el fumar pasivamente es peligroso para la salud, mientras que públicamente han negado que estos peligros estén probados». Ver, BARnes, D.E., HANAUER, P., Environmental Tobacco Smoke: The Brown and Williamson Documents, JAMA, 1995, julio, p. 248.

68 Reproduce literalmente la Rule 23 (a) Federal Rules of Civil Procedure.

69 Idéntica a la Rule 23 (b)(3). 
otros existentes para lograr una sentencia justa y eficiente ${ }^{70}$, en opinión del Tribunal, también se veía satisfecho en el caso Broin ${ }^{71}$. El argumento del Tribunal descansaba en la afirmación de que sería financieramente imposible para los representantes y los miembros del grupo entablar demandas individuales contra los demandados, y por lo tanto, en este supuesto la forma de class action cumple el requisito de «superiority».

A pesar de las estrategias dilatorias planteadas por los abogados de las tabacaleras, el juicio tuvo lugar en un Tribunal de Miami, Estado de Florida. La cantidad indemnizatoria que se reclamaba en concepto de daños era superior a los 5 billones de dólares. El 2 de junio de 1997 comenzó el juicio con la selección del jurado, pero éste se vio afectado por el preacuerdo alcanzado por las tabacaleras con los estados (acuerdo de 20 de junio de 1997). Ante la posibilidad de que el preacuerdo anterior fuese ratificado por el Presidente Clinton ${ }^{72}$, se llegó a un preacuerdo entre los representantes del caso Broin y las tabacaleras. El Tribunal aceptó los términos del acuerdo de negociación por considerarlos justos, razonables y adecuados. Los términos del acuerdo fueron, en líneas generales:

1. Los demandados que han aceptado firmar el acuerdo aprueban el pago de 300 millones de dólares con intereses a una fundación sin beneficios lucrativos. Dicha fundación se creará con la finalidad de realizar investigaciones científicas para la detención y la cura de las enfermedades relacionadas con el consumo de tabaco. Las tabacaleras no tendrán otra relación con la Fundación que no sea la de constituir los fondos económicos.

2. Los demandados acuerdan apoyar la creación de una legislación federal que prohíba fumar en todos los vuelos internacionales que tengan su origen o su destino en los Estados Unidos.

3. Los miembros del grupo, de forma individual, retendrán su derecho a entablar demandas por daños compensatorios o bajo otra causa de pedir distinta de las recogidas en la class action de Broin. No cabrá la interposición de demandas en reclamación de daños punitivos contra los demandados firmantes del acuerdo.

70 Este fue el requisito que el Tribunal de Castano consideró incumplido.

71 "The very purpose of a class suit is to save a multiplicity of suits, to reduce the expense of litigation, to make legal processes more effective and expeditious, and to make available a remedy that would not otherwise exist». Tenney v. City of Miami Beach, 11 So.2d 188, 189 (Fla. 1942).

72 El tema de las demandas entabladas por los estados contra las Tabacaleras por los costes sanitarios lo trataremos más adelante. 
Respecto a los demandados no firmantes quedan vigentes todo tipo de acciones, sin restricción alguna.

4. Para las demandas futuras que, de forma individual, entablen los miembros del grupo sobre Retained Claims, la carga de la prueba de la relación de causalidad del cáncer de pulmón, bronquitis crónica, enfisema, obstrucción pulmonar crónica y sinusitis crónica se trasladará a los demandados firmantes. Para el resto de las enfermedades la carga de la prueba de la relación de causalidad se mantiene en los demandantes.

5. Los demandados firmantes acuerdan abonar los costes «out of pocket» en los que se haya incurrido durante los seis años y medio que ha durado el caso. También acuerdan que abonarán los honorarios de los abogados del grupo, en la cantidad de 46 millones de dólares, siempre y cuando lo apruebe el Tribunal.

6. Los demandados firmantes concluyen que, a pesar de su creencia de que no son responsables de las alegaciones que se hacen en su contra y de que tienen buenas defensas, toman parte en el acuerdo únicamente para evitar mayores gastos, inconvenientes, la carga de personal y de recursos financieros de un proceso complejo y para evitar los riesgos inherentes a un proceso complejo.

No parece que éste sea un acuerdo muy beneficioso para una demanda en la que reclaman 5 billones de dólares. Sin embargo, muchas son las razones que han podido llevar a los representantes del grupo a conformarse con la creación de un fondo de 300 millones y otra serie de medidas. En primer lugar, los representantes del grupo se enfrentaban a la posibilidad de que el Presidente Bill Clinton ratificase el acuerdo alcanzado entre las tabacaleras y los estados. Si éste se hubiera ratificado, la posición jurídica de los componentes del grupo Broin podría verse seriamente afectada. Las tabacaleras aceptaron firmar el acuerdo con los Attorneys General siempre que se les asegurase cierta inmunidad frente a las acciones planteadas contra ellas ${ }^{73}$.

En segundo lugar, los representantes eran conscientes de los graves problemas de prueba a los que se enfrentaban, especialmente en lo relativo a la relación de causalidad. Aunque la Environmental Protection Agency ha declarado el humo de los cigarros como cancerígeno, la es-

73 En un primer momento exigieron una inmunidad absoluta, pero en las negociaciones posteriores aceptaron una inmunidad parcial, dentro de la que se incluye cierta inmunidad hacia las class actions. El acuerdo entre las tabacaleras y los estados no llegó a recibir la aprobación del Congreso. 
trategia de las tabacaleras de cuestionar la relación de causalidad entre la inhalación del humo y las enfermedades pulmonares ha tenido más éxito que las pruebas aportadas por los demandantes. Tal vez fue el miedo a no lograr la prueba de la relación de causalidad lo que les llevó a aceptar una inversión de la carga de la prueba para las demandas futuras basadas en otras causas de pedir. A su favor tenían el que las tabacaleras no podían alegar casi ninguna de las defensas planteadas ante los fumadores activos (asunción de riesgo, compensación de culpas, etc.).

En tercer lugar, los representantes se enfrentaban a la incierta aplicación de la teoría de «market share». La jurisprudencia norteamericana elaboró la teoría de «market share» para aquellos supuestos en los que una vez probada la relación de causalidad, la víctima se encontraba ante la dificultad adicional de probar quién había sido el fabricante concreto del producto que le había causado los daños. Son supuestos en los que múltiples fabricantes ponen en el mercado un producto defectuoso idéntico y la víctima desconoce, o le es casi imposible, probar la marca o el fabricante particular del producto por ella consumido (por ejemplo, fármacos genéricos) $)^{74}$. La teoría se articuló por primera vez en Sindell v. Abbott Laboratories, una class action en la que el Tribunal Supremo de California tuvo que decidir sobre la cuestión. En primer lugar negó las teorías, que habían sido aceptadas por el Tribunal de Apelación de California y otras jurisdicciones, conocidas como «alternative liability» ${ }^{75}$, «concert of action» $\mathrm{e}$ «industry-wide liability». Des-

74 Este tema surgió, por primera vez, en el Tribunal Supremo de California. El producto defectuoso, en este caso, era el DES (Diethylsilbestrol), un estrógeno sintético desarrollado en Inglaterra y recetado en los Estados Unidos desde 1947 hasta 1971 para prevenir el aborto. Las mujeres que lo tomaron no sufrieron daño alguno pero algunas de sus hijas desarrollaron cáncer de útero. Los fabricantes del DES fueron unos trescientos laboratorios farmacéuticos y todos utilizaron la misma fórmula. Los médicos recetaron la droga, normalmente, con su nombre genérico, sin especificar un fabricante concreto. Bajo estas circunstancias, los demandantes eran incapaces de identificar la compañía específica que fabricó las píldoras tomadas por su madre y por ello muchos tribunales declararon que habían fracasado en probar la relación de causalidad (Gray v. United States (S.D. Tex. 1978) 445 F.Supp. 337). Ver, TefF, H., «Market Share» Liability — a Novel Approach to Causation, International and Comparative Law Quarterly, 1982, vol. 31, pp. 840-844.

75 Bajo la doctrina de «alternative liability», cuando dos o más demandados han cometido actos ilícitos en circunstancias que hacen imposible para la víctima la prueba de cuál de ellos causó el daño, la carga de la prueba se traslada a los demandados. Los demandados deben probar que no fueron los responsables del daño (Restatement (Second) of Torts, secc. 433B(3), 1965). Si no lo consiguen, ambos son declarados responsables solidarios. Pero esta solución normalmente se ha adoptado sólo cuando todos los demandados potencialmente responsables han actuado de forma conjunta, como por ejemplo, en el caso clásico Summers v. Tice (33 Cal. $2^{\text {nd }} 80,199$ P. $2^{\text {nd }} 1$ (1948)) en el que dos cazadores simultáneamente dispararon en la dirección de la víctima y sólo uno le causó la lesión. 
pués de analizar las teorías anteriores, el Tribunal procedió a construir una novedosa modificación de la relación de causalidad. Consideró que «era razonable ... medir la probabilidad de que los demandados suministraran el producto que lesionó a la víctima en base al porcentaje de venta de DES de cada uno de ellos». La carga de la prueba se trasladará a cada compañía, que debe probar que no suministró la droga concreta que lesionó al demandante. Si no lo consigue, será responsable por la parte de la indemnización que corresponda a su porción de mercado. La sentencia ha sido criticada por las importantes dificultades administrativas que conlleva la aplicación de la nueva teoría.

Los fumadores pasivos del caso Broin, en el supuesto de que prueben que sus enfermedades pulmonares son debidas a la inhalación del humo del tabaco en los aviones, deberían probar quiénes fueron los fabricantes de los cigarrillos y establecer su tanto por ciento de responsabilidad en la causación de los daños. Si el Tribunal aplica la teoría de «market share», con la inversión de la carga de la prueba que ésta conlleva, la consecución de una sentencia favorable a sus intereses será algo más que una mera posibilidad. Sin embargo, y aunque la teoría tiene muchos adeptos en la doctrina norteamericana, no se aplica de forma uniforme en los tribunales ${ }^{76}$.

El futuro de la class action de Broin y de su preacuerdo es incierto y todavía no ha sido resuelto.

\section{Engle v. R.J. Reynolds Tobacco, et.al}

El fracaso de las class action de Castano tuvo como resultado la aparición de class actions similares pero limitadas en su ámbito territorial. La primera en obtener un veredicto del jurado ha sido la entablada en el estado de Florida, conocida como «caso Engle». La acción se entabló en 1994 por 9 demandantes en beneficio de unos 500.000 residentes del estado de Florida afectados por enfermedades relacionadas con el consumo de tabaco. En la class action se reclaman 200 billones de dólares. Los argumentos jurídicos alegados por los demandantes son los mismos que en el caso Castano: negligencia, strict liability, fraud, etc., y la prueba documental se apoya en los documentos «Brown and Williamson».

El 7 de julio de 1999, un jurado de Florida emitió un veredicto a favor de los demandantes en este caso. En su opinión, las tabacaleras

76 Por ejemplo, los Tribunales Supremos de Wisconsin y de Washington han elaborado una versión modificada de la «market share»: Martin v. Abbott Labs., 102 Wash.2d 581, 689 P.2d 368 (1984); Collins v. Eli Lilly Co., 116 Wis.2d 166, 342 N.W.2d 37 (1984). 
eran responsables de las enfermedades de miles de fumadores, ya que la industria fabricó y vendió de forma consciente un producto defectuoso que causa cáncer de pulmón y otras enfermedades.

Ahora el jurado debe afrontar la segunda fase del juicio; la determinación de las indemnizaciones económicas que corresponden a los demandantes. Los demandados alegan que no existe un prototipo de víctima, puesto que los fumadores sufren enfermedades distintas. Por ejemplo, Engle padece de asma crónica, Lacey (otro de los demandantes) ha sufrido amputación de ambas piernas como consecuencia de un problema circulatorio conocido como la enfermedad de Buerger, Angell (también demandante) padece cáncer de garganta. La estrategia de los demandantes es la de negar la certificación del grupo como class action debido a la imposibilidad de establecer las indemnizaciones económicas que corresponden a los miembros del grupo. Edward Moss, abogado de las tabacaleras, ha declarado:

"What we have here are several individuals who suffer from a whole bunch of diseases that are allegedly tied to smoking. But there are a whole bunch of other things that could have caused these individual illnesses. These people have smoked different amounts of cigaretes, have different genetic factors, have worked in different places, been exposed to different substances and have had varying health histories, all of which may have contributed to their different diseases. These are individual issues that should be treated as individual cases, not as a class action.»

El futuro de esta class action, a pesar de la gran atención que ha recibido por parte de los medios de comunicación es incierto, porque, aunque supere la segunda fase del juicio, las tabacaleras han anunciado su intención de apelar ante los tribunales superiores. Si el jurado llega a un veredicto el quantum de la indemnización será el mayor concedido jamás en una acción contra las tabacaleras, lo que, evidentemente, estimulará al resto de las acciones pendientes en otros estados. ¿Por qué la industria del tabaco no ha buscado un acuerdo extrajudicial con la class action como ya logró en el caso Broin? Según Mark Gottlieb, abogado de la Tobacco Liability Project en la Northeastern University School of Law:

«Si la industria negocia este caso, básicamente estaría aceptando su responsabilidad por las lesiones sufridas por todos los fumadores. Estaría enviando el mensaje de que se termina la batalla y de que desea indemnizar las lesiones sufridas por los consumidores. No parece que esto vaya a ocurrir por ahora.» 


\section{Demandas entabladas por los estados (Attorneys General)}

Un tercer grupo de demandas contra las tabacaleras lo componen las demandas de los Attorney General de los estados en reclamación de los costes de asistencia médica (Medicaid ${ }^{77}$ ) de las enfermedades causadas por el uso o consumo del tabaco fabricado por los demandados ${ }^{78}$. La idea de que el Gobierno recobre estos gastos no es nueva ${ }^{79}$, si bien sólo a partir de 1994 se han llevado a cabo acciones gubernamentales contra la industria del tabaco. Las medidas que han adoptado los Gobiernos estatales y el Gobierno federal son diferentes, pero existen muchas similitudes en la forma en la que se enfrentan al problema.

El estado que inició la campaña contra las tabacaleras fue Florida, que marcó con su actuación las pautas posteriores. En un primer momento, con el fin de evitar los problemas a los que se habían enfrentado los particulares y las class actions en sus demandas, Florida reformó la Medicaid Third-Party Liability Act estableciendo una nueva acción por la que el estado puede demandar por sus gastos de Medicaid relacionados con el tabaco ${ }^{80}$. Las tres provisiones más importantes de esta Ley son: abolición de las defensas del common law de asunción del riesgo y de negligencia contributiva, permitir el uso de estadístivas en la prueba de la relación de causalidad y del daño, y aplicación de la teoría de «market share liability».

El estado de Florida observó que, como este proceso no fija su atención en el individuo particular y en la marca de tabaco que éste fumaba, el mecanismo de «market share» permite al estado demandar a

77 BALCK, op. cit., p. 678:

«Medicaid: A form of public assistance sponsored jointly by the federal and state governments providing medical aid for people whose income falls bellow a certain level.»

${ }^{78}$ La Universidad de California y el Center for Disease Control elaboraron un informe (Current Trends: Medical-Care Expenditures Attributable to Cigarette Smoking, United States, Morbidity \& Mortality Wkly.Rep., 1994, vol 43, p. 469) del que se desprendía que de los 308,7 billones de dólares empleados en «cuidados médicos directos» en 1987, 21,9 billones (el 7,1\%) correspondían a gastos derivados del consumo de tabaco. El estudio estimó que en 1993 un total de 50 billones de dólares se gastó para tratar las enfermedades relacionadas con el tabaco. Sin embargo, hay otras voces que afirman que los gastos son menores porque los fumadores ya abonan parte de ellos con los impuestos sobre el tabaco.

El estudio también informa sobre la cantidad de dinero abonada por la Medicaid, Medicare y otras fuentes públicas en 1987: la cantidad asciende a 9,512 billones; esta cantidad se eleva a 21,36 billones en 1993.

79 Ver, por ejemplo, Donald W. GANer, Cigarettes and Welfare Reform, Emory Law Journal, 1977, vol. 26, pp. 264 y ss.

80 Fla.Stat.Ann. 409.910 (West 1996 \& Supp. 1996). 
todas las firmas más importantes y recibir indemnización en base a su parte de mercado, sin necesidad de probar la marca de tabaco consumida por cada fumador del estado de Florida. Además, se afirma que los fumadores individuales pueden haber asumido el riesgo de fumar, o contribuido a su enfermedad, con su propia negligencia pero el estado no ha asumido ese riesgo y no fue negligente en la transacción entre el fabricante y el consumidor, por lo que no se pueden aplicar las teorías de asunción de riesgo y de negligencia o culpa comparativa ${ }^{81}$. La Ley también recoge que el estado no necesita identificar a los beneficiarios de la Medicaid que sufrieron daños por el consumo de tabaco. Es suficiente, para establecer la existencia y la magnitud del daño, la prueba estadística de la mortalidad o lesividad del consumo de tabaco en el estado de Florida.

El segundo paso fue la interposición de la demanda contra todas las tabacaleras y contra algunas instituciones relacionadas con ellas, por ejemplo The Council for Tobacco Research-U.S.A. Inc. ${ }^{82}$.

La actuación del estado de Florida supuso el comienzo de un gran número de demandas (más de 40). Los Attorneys General de otros esta$\operatorname{dos}^{83}$ siguieron su ejemplo y en líneas generales las demandas se basan en las teorías de:

1. NEGLIGENCE: Los demandados tenían la obligación de ejercer un cuidado razonable en la fabricación, venta y/o distribución de sus cigarros. Los demandados incumplieron esa obligación. Como consecuencia de este incumplimiento los cigarros se fabricaron, vendieron y distribuyeron en el estado. Los beneficiarios de Medicaid contrajeron enfermedades por el uso de los cigarros y el estado se vio obligado a proveer de asistencia médica a dichos beneficiarios.

81 Respecto a los honorarios de los abogados se establece un sistema según el cual recibirán el $25 \%$ de cualquier veredicto que se consiga. Con esta medida se busca la colaboración de despachos de abogados independientes y ahorrar dinero al estado de Florida en el supuesto de que la demanda no tenga éxito.

${ }^{82}$ Las compañías demandadas son:

La American Tobacco Company, American Brands, Inc., Reynolds Tobacco Company, RJR Nabisco, Industries PLC, British American Tobacco Co., Batus Holding, Inc., Brown \& Williamson Tobacco Corporation, Philip Morris Companies, Philip Morris Incorporated, Brooke Group, Brooke Group LTD., Liggett Group, Inc., Liggett \& Meyers, Inc., Loews Corporation, Lorillard Corporation, United States Tobacco Company, The Council for Tobacco Research-U.S.A. Inc., Tobacco Institute, Inc., Hill \& Knowlton, Inc., Dosal Tobacco Corp.

83 Ver, Health Care Reform: States Rush to File Medicaid Tobacco Suits, Wash.Health Wk., 1996, 3 de junio. 
2. STRICT LIABILITY FOR DEFECTIVE AND UNREASONABLY DANGEROUS PRODUCT: Como resultado directo y próximo de un defecto de diseño, de fabricación, y de información, los cigarros de los demandados fueron y son en sí mismos defectuosos e irrazonablemente peligrosos. La condición defectuosa de los cigarros es la causa próxima y directa que causó que miles de residentes del estado sufriesen enfermedades relacionadas con el consumo de tabajo y son la causa próxima y directa de que el estado abonase millones de dólares para el cuidado sanitario necesario a estos residentes.

3. BREACH OF EXPRESS AND/OR IMPLIED WARRANTIES: Las tabacaleras han incumplido la «implied warranty» porque sus productos no cumplen con sus propósitos intencionados.

4. NEGLIGENCIA PERFORMANCE OF A VOLUNTARY UNDERTAKING: Las tabacaleras voluntariamente asumieron la obligación y la responsabilidad de informar honestamente de todas las investigaciones en relación al fumar y a la salud mediante sus pronunciamientos públicos. Los demandados incumplieron sus obligaciones, no sólo por el fracaso de informar de esas investigaciones, sino por publicar activamente y con conocimiento una ciencia fraudulenta. Además, los demandados incumplieron su obligación al suprimir las investigaciones negativas en relación al tabaco y la salud. Los demandados sabían o debían saber que los fumadores, los demandantes y las regulaciones del Gobierno descansarían en sus pronunciamientos.

5. FRAUD, INTENTIONAL MISREPRESENTATION: LOS demandados intencionadamente suprimieron material sobre el peligro de fumar. Los demandados participaron en anuncios de cigarros que los representaban, al menos, como poco dañinos y casi como saludables, ese retrato fue una misrepresentation intencional de la naturaleza peligrosa de los cigarros. El propósito de la supresión de los datos negativos era el de confundir a los consumidores potenciales sobre los peligros de los cigarros incentivándoles a fumar.

6. CONSPIRANCY AND CONCERT OF ACTION: Los demandados llegaron a un acuerdo para suprimir la información científica y médica en relación al fumar y sus enfermedades. Participaron y cooperaron entre ellas en la conspiración anterior. Para llevar a cabo esta conspiración crearon el Tobacco Institute y el Council of Tobacco Research. Ambos institutos participaron activamente en la conspiración. Como consecuencia, los demandados pudieron continuar vendiendo sus cigarros y confundiendo al público. 
En el ámbito federal, los senadores Frank Lautenberg y Tom Harkin propusieron al Congreso un proyecto de ley similar a la del estado de Florida, aunque sin éxito ${ }^{84}$.

Las tabacaleras respondieron con la interposición de demandas para lograr la declaración de inconstitucionalidad de las leyes y de las demandas entabladas en su contra por esta nueva causa de pedir. En ellas, se afirma que la aplicación retroactiva de la Ley, la abolición de las defensas de asunción de riesgo y negligencia contributiva, la aplicación de la teoría de market share, y el uso de estadísticas como prueba suficiente son inconstitucionales. La respuesta de los tribunales, hasta el momento, ha sido la de declarar que las leyes aprobadas por los estados no pueden tener efectos retroactivos; ha limitado el uso de las estadísticas, pero ha mantenido el resto de las provisiones ${ }^{85}$.

Las tabacaleras, en especial R.J. Reynolds, han contestado a las demandas afirmando que no existe causa de pedir porque los estados «permitieron, regularon, facilitaron y obtuvieron beneficios de la venta de los cigarros». En realidad, lo que R.J. Reynolds está buscando es que se aplique a los estados la teoría de «asunción de riesgo», que tanto éxito les ha proporcionado como defensa en demandas pasadas. Asimismo, consideran que en el caso de que se conceda una indemnización ésta deberá moderarse de acuerdo con el impuesto pagado al estado como resultado de la venta de tabaco.

El éxito de las demandas entabladas por los estados no parece que vaya a estar en la obtención de sentencias a su favor. Su éxito se encuentra en que han obligado a la industria tabaquera a entablar negociaciones y a abandonar su clásica postura de defender en los tribunales todas las demandas en su contra ${ }^{86}$.

84 S. 2245 , 103d Cong. 2d Sess. (1994).

85 En apelación, el Tribunal Supremo de Florida (Agency for Health Care Admin. v. Associated Indus. of Fla., Inc., 678 So.2d 1239, 1257 (Fla. 1996)), declaró que la Ley no era «ni arbitraria, ni caprichosa. Fue la respuesta racional a una necesidad pública». El Tribunal Supremo también negó la alegación de las tabacaleras de que la abolición de las defensas convertía a la ley en inconstitucional, y dijo que el uso de estadísticas era totalmente válido y podía ser ejercitado por el poder del Parlamento. Sin embargo, no toda la decisión del Tribunal Supremo favorecía las pretensiones del estado de Florida. El Tribunal Supremo estableció que Florida debe identificar a cada beneficiario individual de Medicaid por el que se pide el reembolso. Mantuvo la decisión de que sólo se podía pedir indemnización después del 1 de julio de 1994. Finalmente, el Tribunal Supremo dijo que se podía pedir reclamación bajo la teoría de responsabilidad solidaria o bajo la de «market share», pero que no se podían aplicar las dos teorías de forma simultánea.

86 En agosto de 1997, el estado de Florida llegó a un acuerdo con las tabacaleras para terminar con la demanda planteada. También se han negociado las demandas de Texas y de Mississippi. Se recogen más adelante. 


\section{Acuerdos extrajudiciales}

Junto con los juicios por los costes de Medicaid, los entablados por particulares y las distintas class actions, la industria del tabaco se enfrenta a los proyectos de la FDA (Food and Drug Administration) sobre regulación de la venta y publicidad del tabaco ${ }^{87}$ y a la investigación de la división criminal del Departamento de Justicia por fraude ${ }^{88}$. Esta guerra con múltiples frentes ha hecho que las tabacaleras se planteen la opción de seguir defendiéndose contra las demandas y recurriendo los proyectos de la $\mathrm{FDA}^{89}$, o bien de tomar una solución alternativa. El precio de la primera opción es alto - aunque las tabacaleras no han revelado el coste de sus defensas, se cree que alcanza la cifra de $600 \mathrm{mi}-$ llones anuales ${ }^{90}$ — por lo que la segunda opción, la negociación, cobra cada día más peso como solución del problema.

87 El 26 de agosto de 1996, el Presidente Clinton declaró a la nicotina como producto adictivo. Esta medida implicaba que el tabaco, que hasta ese momento había sido considerado como "producto agrícola» y por lo tanto sometido a las regulaciones de los productos agrícolas, dejaba de serlo, y se le daba así poder a la Food and Drug Administration para regular el tabaco (originariamente la nicotina se encontraba dentro del listado de drogas realizado por la FDA, pero, como consecuencia de los esfuerzos de la industria del tabaco, se retiró de la lista).

La FDA ha elaborado una propuesta de normativa especialmente encaminada a prohibir la venta de tabaco a menores. En ella se recogen medidas como la de prohibir la colocación de máquinas expendedoras de tabaco en locales a los que tengan acceso los menores, la obligación de mostrar un documento acreditativo de la edad para la compra de tabaco, prohibir el patrocinio de eventos deportivos por parte de las tabacaleras y otras medidas que limitaban seriamente la publicidad de tabaco.

Regulations Restricting the Sale and Distribution of Cigarettes and Smokeless Tobacco to Protect Children and Adolescents, 61 Fed. Reg. 44,396 (1996) (to be codified at 21 C.F.R. $\$ \$ 801,803,804,807,820$ and 897).

88 Ver Thomas, P., Schwartz, J., U.S. Widens Tobacco Investigation: Justice Department Pressures Mid-Level Workers in Fraud Probe, Washington Post, 1996, 8 de septiembre, en A-1.

89 En respuesta a la propuesta de normativa sobre producción y publicidad de tabaco, las tabacaleras (incluido el grupo Liggett) interpusieron, el 10 de febrero de 1997, una demanda ante la jurisdicción federal con el fin de impedir su entrada en vigor. La industria alega que el Congreso de los Estados Unidos nunca ha dado poder a la FDA para elaborar normativa alguna sobre el tabaco, por lo que carece de jurisdicción. Además, el tabaco no puede ser considerado como una droga o fármaco de los definidos en la Food, Drug, and Cosmetc Act. Por último, también alegan que las restricciones publicitarias que se contienen en la propuesta de normativa violan la 1. ${ }^{\mathrm{a}}$ enmienda de la Constitución de los Estados Unidos, por ser unas medidas desmedidas y porque existen otros medios con los que FDA puede reducir el consumo de tabaco en menores sin limitar la publicidad dirigida a adultos.

90 Plummer, D., Billions at Stake as Lawsuits Force Showdown on Smoking, Atlanta J. \& Constitution, 1996, 15 de septiembre, en D-5. 
El primer preacuerdo se llevó a cabo entre el grupo Liggett ${ }^{91}$ y los integrantes de la class action de Castano en 1996. Dos días después se alcanzó un preacuerdo entre Liggett y 5 estados que habían demandado a la industria del tabaco para recuperar costes sanitarios ${ }^{92}$. Sin embargo, el resto de las tabacaleras decidió continuar con su defensa en los tribunales. A partir de esa fecha las demandas de los estados se sucedieron y el grupo Liggett decidió negociar un acuerdo más amplio que los abarcase a todos. El 20 de marzo de 1997, después de muchas negociaciones, se llegó a un preacuerdo que contenía concesiones sin precedentes; por ejemplo, la entrega de documentos que hasta entonces se habían considerado secretos (pero cuya existencia todo el mundo conocía; los documentos Brown \& Williamson) y el acuerdo de que Liggett permitiría a sus empleados —incluso a sus abogados- testificar en juicio contra el resto de la industria. Además, Liggett se convirtió en la primera tabacalera que admitía los peligros para la salud del consumo de tabaco y la naturaleza adictiva de la nicotina ${ }^{93}$ (aceptó incluir en las etiquetas de sus cajetillas de tabaco un aviso sobre los peligros de la nicotina).

91 El preacuerdo se firmó con Brooke Group Ltd., Liggett \& Myers Inc. y Liggett Group Inc. Nunca llegó a firmarse el acuerdo definitivo.

${ }_{92}$ Los estados eran: Florida, Massachusetts, Minnesota, Mississippi y West Virginia. En este preacuerdo inicial, el grupo Liggett, que no admitía culpabilidad alguna, se comprometía a abonar durante los siguientes 25 años una porción de sus beneficios que se destinarían a programas para cesar en el consumo de tabaco. Los pagos ascenderían al 5\% de sus ingresos antes de impuestos; anualmente, y durante 25 años. El máximo anual sería de 50 millones de dólares al año o de 1,25 billones de dólares durante todo el periodo.

93 Las obligaciones contraídas por Liggett eran:

- Obligaciones económicas. Un pago inicial de 25 millones de dólares y durante 25 años pagos equivalentes al 25\% de los beneficios de Liggett antes de impuestos.

— Cambio en el etiquetaje de sus cajetillas de tabaco: «Fumar cigarros causa adicción».

- Cumplir las obligaciones de la Food and Drug Administration.

- Colaborar con los estados en las demandas que éstos planteen o tengan planteadas contra la industria del tabaco.

— Realizar una declaración pública: El Director de Liggett, Bennett S. LeBow, se comprometió a hacer una declaración pública, en la que se dijo que ellos estaban al corriente de que el consumo de tabaco causa problemas para la salud (como cáncer, problemas cardiovasculares de corazón y enfisema) y también de la naturaleza adictiva de la nicotina.

- Retirar todas las acciones que tenga planteadas contra los estados firmantes.

Las obligaciones de la otra parte eran: retirar todas las acciones que tuvieran planteadas contra Liggett, y colaborar activamente para que la futura legislación del Congreso reflejase la cooperación del Grupo. 
Por primera vez se rompía el frente común que durante más de treinta años había presentado la industria del tabaco ${ }^{94}$, y en el mes de abril comenzaron las negociaciones entre la industria tabaquera y los Attorneys General de 40 estados, bajo la atenta mirada de la Casa Blanca ${ }^{95}$. En junio se anunció un principio de acuerdo en el que las tabacaleras aceptarían pagar en los siguientes 25 años un total de 368,5 billones de dólares ${ }^{96}$ destinados a compensar los daños causados por el tabaco, a la financiación de programas de educación para dejar de fumar, y al recorte de la publicidad de sus productos. A cambio, la industria conseguiría una inmunidad parcial frente a las demandas de los particulares. Una de las concesiones más grandes hechas por la industria es el reconocimiento de que la FDA tiene poder para regular el tabaco, incluyendo la posibilidad de que reduzca los niveles de nicotina de los cigarrillos, y prohibir la venta de productos que contengan nicotina a menores de 12 años. El preacuerdo también requiere que la industria entregue a la FDA todas las investigaciones realizadas sobre el tabaco. La aprobación del convenio por el Presidente Clinton daría lugar a la creación de una ley federal en la que se recogería su contenido. A pesar de que Clinton anunció que en el plazo de un mes emitiría un dictamen, no lo hizo. El Congreso recibió el preacuerdo con cautela y la mayoría del partido republicano manifestó que un acuerdo negociado fuera de los confines del Congreso debía ser considerado sólo como una serie de recomendaciones, por lo que para ellos su importancia era relativa ${ }^{97}$.

Mientras tanto los estados de Florida y Mississippi negociaron sus demandas de Medicaid con éxito y alcanzaron sendos acuerdos. Florida logró, entre otras medidas, que la industria tabaquera se comprometiera a pagar 1,7 billones de pesetas y Mississippi logró una indemnización de medio billón de pesetas ${ }^{98}$.

94 El mismo día en que se firmó el acuerdo, el resto de la industria consiguió una orden de un Tribunal de North Carolina para impedir que Liggett entregara los documentos por estar éstos protegidos por el privilegio de abogado-cliente. Sin embargo, si los documentos contienen pruebas de una conducta criminal no estarán protegidos por el privilegio de abogado-cliente y podrán ser utilizados como prueba.

95 El Secretario de prensa de la Casa Blanca dijo que estaban escuchando de cerca las negociaciones pero que no eran parte directa de ellas.

96 Los «billones de dólares» recogidos en este artículo hacen referencia a «billón» como igual a mil millones y no como equivalente a 1 millón de millones.

97 El portavoz de la House of Representatives, New Gingrich, dijo a la Agencia Reuters: «La Constitución no contiene la posibilidad de que el Congreso delegue su poder legislativo a un grupo privado en una reunión secreta».

98 El acuerdo alcanzado por el estado de Florida se encuentra en INTERNET, http://stic.neu.edu/Fl/flsettle.htm.; el acuerdo de Texas se puede encontrar en http://stic.neu. edu/Tx/Texas-settlement.htm. Texas y Minnesota también llegaron a acuerdos similares. 
Comentario sobre el preacuerdo de junio de 1997

Algunos de los puntos más destacados del acuerdo son:

- La industria del tabaco pagaría 368,5 billones de dólares, durante 25 años, a un fondo del que se extraerían los recursos necesarios para indemnizar las demandas por daños a las personas y las de los programas de sanidad pública (divididos en un pago inicial en el momento en que se apruebe la ley de 10 billones de dólares y pagos anuales que irán de 8,5 billones a 15 billones) ${ }^{99}$. Cerca de 308 billones de dólares se destinarían a negociar las demandas y 60 billones se destinarían a las indemnizaciones punitivas. El pago anual al que se obligaba a la industria debería ajustarse anualmente a la inflación en un 3\% anual.

- La cantidad total que la industria del tabaco tendría que abonar anualmente para negociar las demandas entabladas estaría sometida a un límite máximo anual de 5 billones de dólares.

- La industria tabaquera reconocería, formalmente, que la Food and Drug Administration tiene poder normativo sobre el tabaco y podría, eventualmente, prohibir la utilización de nicotina.

99 Sumamry of Settlement Provision, p. 3638:

Of the $\$ 368.5$ billion payments that will be received from the tobacco companies over twenty five years, the Agreement recommends that approximately $\$ 93$ billion, or roughly $25 \%$ of the total payments, be used to fund public health programs, as follows:

- HHS will receive \$125 million for the first three years, and \$225 million annually thereafter to fund youth preventio, adult cessation, research and other programs.

-FDA will receive \$300 million annually to carry out its obligations ant to enforce the provisions of this settlement.

- State and local governments will receive $\$ 75$ million for the first two years, $\$ 100$ million the third year, and $\$ 125$ million annually thereafter to fund community based prevention programs.

— \$100 million per year will be dedicated to fund research and development of tobacco prevention and cessation methods.

- Sports teams and events that lose tobacco industry sponsorship will receive \$75 million per year for the first 10 years following the effective date of the settlement. After 10 years, these funds will be reallocated to other public health programs.

-An independent, nonprofit organization to be formed will receive $\$ 500$ million per year to fund multimedia public education campaigns.

-A newly formed Tobacco Cessation Trust Fund will receive \$1 billion per year for the first four years and $\$ 1.5$ billion per year thereafter. The fund, which will be managed by the Secretary of HHS, will be used to assist existing smokers in their efforts to quit smoking.

-A Public Health Trust Fund under the control of a Presidential Commission will receive $\$ 25$ billion of fund specific tobacco related medical research. Representatives from the public health community and the state Attorneys General will serve on the Commission. 
Transcurridos 12 años desde la entrada en vigor del acuerdo, la agencia podría prohibir la utilización de nicotina totalmente.

- Las tabacaleras acordaron importantes restricciones en la publicidad que pudiese llegar a menores. Las restricciones comprenden desde la eliminación de caricaturas o de imágenes humanas en los paquetes de tabaco, a la prohibición de carteles y vallas publicitarias.

- La industria acordó la creación de un programa público de ámbito nacional para la educación contra el consumo de tabaco.

- La industria acordó un cambio en las etiquetas de sus productos; la información ocuparía el $25 \%$ del paquete. La etiqueta incorporaría un nuevo lenguaje en el que se avisase de que el consumo de cigarrillos es adictivo y que el humo que desprenden causa cáncer en los no fumadores.

- Se acuerda la imposición de multas a las tabacaleras si el consumo de tabaco entre los menores no desciende en un $42 \%$ en 5 años, $58 \%$ en 7 años y $67 \%$ en 10 años.

- La industria acordó la creación de programas que ayuden a dejar de fumar y a proveer gratuitamente de las medidas necesarias para que puedan abandonar el tabaco quienes carecen de medios.

1. Cuando el preacuerdo fue alcanzado, todas las partes convinieron en que era necesario presentar un frente común en el Congreso para lograr la creación de la ley estatal que ratificara sus contenidos. El poder de las tabacaleras en el Congreso es grande, por lo que la aceptación de la industria era necesaria para lograr que la ley fuese aprobada ${ }^{100}$. La aprobación de una ley federal se consideraba una victoria, porque la industria tabaquera nunca había perdido un caso de reclamación de costes sanitarios en los tribunales y los Attorneys General decidieron que era mejor obtener una victoria parcial en el Congreso que enfrentarse a los riesgos que suponen los tribunales. Este estado de opinión fue cambiando con el paso del tiempo y algunas de las partes del preacuerdo se negaron a su aprobación en la forma inicialmente adoptada. La creencia de los Attorneys General de que la industria del tabaco no negociaría las demandas de Medicaid de forma individual se ha demostrado falsa. La industria ha llegado a

100 En 1995-1996, Philip Morris contribuyó a la campaña electoral con 2.520 .518 dólares para el partido republicano y con 496.518 dólares para el partido demócrata. En total se contribuyó con más de 5 millones de dólares para los republicanos y 1 millón para los demócratas. Ver, Abramson J., Meier B. Tobacco Bracedfor Costly Fight. The New York Times, New York City, december 15, 1997. 
acuerdos en Mississippi ${ }^{101}$, Florida ${ }^{102}$, Minnesota y Texas ${ }^{103}$ en términos más favorables que los del acuerdo global, así como en un caso de San Francisco contra la publicidad de Joe Camel ${ }^{104}$ y la class action de Broin ${ }^{105}$.

2. A favor de la adopción del preacuerdo también estaba el miedo a una posible declaración de quiebra de alguna, o algunas, de las tabacaleras. La posibilidad sigue existiendo. Los que apoyan el preacuerdo argumentaban que éste era necesario porque asegura la continuidad financiera y evitaba la declaración de quiebra de las tabacaleras. No hay duda de que las declaraciones de quiebra cambiarían las reglas de juego actuales y harían más difícil que los créditos actuales y los potenciales fuesen abonados. Sin embargo, lo que no es probable es que la industria del tabaco se viese favorecida. Los que se opusieron a la adopción del acuerdo afirmaban que la posibilidad de una declaración de quiebra en una o más tabacaleras debía valorarse de forma cuidadosa, pero no debía ser temida y no era razón suficiente para adoptar un mal acuerdo.

La motivación que subyace en el derecho de quiebra de los Estados Unidos es doble. Primero, asegurar una equitativa distribución de los créditos. Segundo, este derecho concede un «fresh start» para el deudor. Una vez que el deudor satisface sus deudas podrá comenzar con su actividad de nuevo. Una de las ventajas de la declaración de quiebra es que el deudor gana tiempo. Las formas más comunes de una declaración de quiebra voluntaria son las del capítulo $7^{106}$ y 11 del Bankruptcy Code. Los dos procedimientos se inician en el Bankruptcy Court, donde el deudor solicita la declaración de quiebra para su empresa.

Bajo el capítulo 11, el deudor junto con el Bankruptcy Court y los acreedores elaboran un «plan de reorganización» según el cual la em-

101 State of Mississippi Tobacco litigation in the Chancery Court of Jackson Country, Mississippi Cause No. 94-1429, Memorandum of Understanding July 2, 1997.

102 Florida v. American Tobacco Co., Case No. 95-1466 AH, Court of the Fifteenth Judicial Circuit, Palm Beach Florida, Settlement Agreement 8/25/97.

103 Texas v. American Tobacco Co., Case No. 5-96CV-91, USDC E.D. Texas Comprehensive Agreement and Release 1/16/98.

104 Janet C. Mangini v. R.J. Reynolds Tobacco Co, Case No. 939359, Superior Court of the State of California Country of San Francisco, Settlement and Consolidation Agreement 9/8/97.

105 Norma R. Broin v. Philip Morris, Case No. 91-49738, Circuit Court of the Eleventh Judicial Circuit, Dade County Florida, Settlement Agreement, 10/9/97.

106 Bajo el capítulo 7 el resultado de la quiebra es la liquidación de todos los bienes. Los bienes de la empresa se venden, el producto de esta venta se entrega a los deudores en orden a la prioridad de los créditos recogida en el Bankruptcy Code y la empresa cesa en su actividad. 
presa deudora cumplirá con sus créditos en la manera aceptada por todas las partes. La finalidad del capítulo 11 es la de reorganizar la empresa de tal forma que sea capaz de cumplir con sus deudas pasadas y futuras.

Es posible que como consecuencia de los procesos presentes y futuros contra las tabacaleras alguna compañía solicite una declaración de quiebra en base al capítulo 11. La tabacalera podría hacerlo antes de que tuviese un veredicto en su contra, porque para que comience el procedimiento del capítulo 11 no es necesario que el deudor sea insolvente en el momento de iniciarse éste. Existen tres ejemplos históricos: la quiebra de los fabricantes de asbesto, de Dalkon Shield y la de los fabricantes de implantes de pecho de silicona ${ }^{107}$. Basándose en los precedentes anteriores existe una probabilidad razonable de que algunas tabacaleras busquen la declaración de quiebra. Aunque para que se declare la quiebra se exige el requisito de «buena fe» (la empresa no puede buscar con la declaración de quiebra, únicamente, el evitar las deudas o los procesos judiciales en su contra), la magnitud de las demandas contra las tabacaleras sugiere que el requisito de buena fe no presentaría grandes dificultades ${ }^{108}$.

Una de las principales motivaciones de una tabacalera para iniciar el procedimiento de quiebra del capítulo 11 pudiera ser la de lograr que sus costes y sus responsabilidades sean predecibles y manejables. Un valor adicional podría ser la reducción a la exposición pública negativa a la que constantemente se ven expuestas como resultado de los procesos. Sin embargo, también tendría efectos negativos serios. Una declaración de quiebra enfrentaría a la empresa a un gran número de riesgos. Por ejemplo, teniendo en cuenta la magnitud de la responsabilidad potencial a la que se enfrentan las tabacaleras no está claro que se aprobase un «plan de reorganización» si la empresa continúa fabricando y vendiendo tabaco. Según estudios, el daño global causado por la industria tabaquera anualmente se estima en 100 billones de dólares. Si el Bankruptcy Court aprueba un plan en el que se establezca un fondo de depósito que reembolsase a las víctimas la mitad del daño, las tabacale-

107 Por ejemplo, en Johns-Manville Corp., 36 B.R. 743 (Bankr. S.D.N.Y. 1984) se recurrió al capítulo 11, aún cuando era una empresa viable financieramente (un billón de dólares). El problema era doble: los procesos entablados contra la empresa podían exponerla al pago de unas indemnizaciones superiores a 5 billones de dólares, más el coste de defender dichas demandas. El Tribunal permitió la declaración de quiebra e incluyó como deudores a los «demandantes futuros».

108 Debido a las solventes finanzas de las tabacaleras, éstas tendrán que recurrir a la magnitud y al número de demandas planteadas en su contra, en aras a lograr una declaración de quiebra del capítulo 11. 
ras tendrían que financiar 50 billones anuales. Esta cantidad es superior a los beneficios teóricos de las compañías tabaqueras, y tendría como resultado que la quiebra fuese la del capítulo $7 \mathrm{y}$ no la del capítulo $11^{109}$. Un segundo argumento, en contra de la quiebra, es el de que el plan de reorganización podría exigir a las empresas un cambio fundamental en sus planteamientos para evitar futuras responsabilidades. El Bankruptcy Court y los acreedores podrían argumentar que el tabaco es inherentemente peligroso y exigir que se cesase o se limitase seriamente la fabricación del producto que ha dado lugar a las demandas (en los tres ejemplos clásicos —asbesto, Dalkon Shield e implantes de pecho de silicona- la empresa tuvo que cesar o restringir la fabricación del producto). Es probable que un plan de reorganización exigiese mayores cambios en el comportamiento de la industria que los propuestos en el acuerdo global. Un tercer argumento se encuentra en que, aunque la regla general es que una vez concedida la declaración de quiebra por el tribunal al deudor se le protege de las demandas y deudas que tenga contraídas, existen excepciones; por ejemplo, si los intereses de una de las partes no están protegidos en la quiebra. Además, la declaración de quiebra reduciría el valor de la compañía afectando a su viabilidad financiera y al valor de sus acciones. Por todo ello, los que se oponen a la firma del acuerdo actual opinan que no parece que el miedo a una declaración de quiebra sea tan real como se apuntó en el momento de la negociación ${ }^{110}$.

3. Cuando en junio de 1997 se alcanzó el preacuerdo, una de las partes que más llamó la atención fue la cantidad económica que las tabacaleras tenían que entregar a los distintos fondos (368,5 billones de dólares). El éxito inicial de los Attorneys General se vio cuestionado por estudios que alegaban que dichas cantidades eran más rentables para la industria tabaquera que para los programas de salud de los estados. Raramente los acuerdos extrajudiciales compensan a las víctimas el $100 \%$ de sus daños; se acepta una indemnización menor para evitar los riesgos que conlleva el sistema judicial. Sin embargo, no sólo se cuestionaba que la cantidad que recibirían los demandantes era muy inferior a la que les concederían los tribunales — si tuvieran éxito en sus demandas-, sino que también se cuestionaba que las fuentes que abas-

109 Ver, Fox, B., Lightwood, J.M., Glantz, S.A., A Public Health Analysis of the Porposed Resolution of the United States Tobacco Litigation, Internet, (http:/www.library. ucsf.edu/tobacco/ustl/), 1988, febrero.

110 Los que apoyan el acuerdo defienden que éste aseguraría que todas las deudas recogidas en él tendrían prioridad y que las medidas recogidas en aras a lograr planes anti-tabaco y otras medidas protectoras nunca se lograrán en una quiebra. 
tecerían el fondo (de donde se extraerían dichas cantidades indemnizatorias) fueran a ser suficientes. En su opinión, la cantidad económica del preacuerdo era enorme en términos absolutos pero pequeña si se compara con el daño que causa el tabaco. Aun cuando se contemple desde un punto de vista más limitado, es decir, que el propósito del preacuerdo era el de reembolsar a los estados por los gastos de Medicaid futuros junto con la creación de programas específicos de sanidad públicos, los pagos no eran suficientes para cumplir ambos propósitos ${ }^{111}$.

4. Las críticas hacia el sistema de pagos establecido en el preacuerdo hacían referencia también a la deducción de impuestos ${ }^{112}$ :

«The settlement will permit tobacco companies to deduct these expenses from their federal income tax liabilities as ordinary and necessary business expenses.»

Todos los pagos que realice la industria de tabaco, incluyendo los que correspondan a daños punitivos, eran deducibles de impuestos. La deducción daría lugar a que el impacto económico del convenio en la industria tabaquera se viese reducido y a que se traslade el coste a los contribuyentes norteamericanos. El Gobieno federal recibiría menos ingresos por impuestos sobre el tabaco. De tal forma que parte de los pagos de las tabacaleras se transferirían de la industria tabacalera al Gobierno federal, transformando el preacuerdo en un programa financiado federalmente ${ }^{113}$.

111 Vid., A. Public Health Analysis of the Porposed Resolution, loc. cit.:

"If the deal is designed to reimburse for all damage done by tobacco, it provides less than 10 cents on the dollar.»

En este informe se aprecia también que el fondo establecido en el acuerdo para abonar las responsabilidades civiles es claramente insuficiente:

"The civil liability fund is inadequate to cover even the medical costs and lost wages associated with tobacco use. The private sector bears approximately $\$ 70$ billion dollars in medical costs and lost wages due to tobacco; these damages are potentially recoverable damages by potential private plaintiffs every year. At its maximum the civil liability fund will only provide $\$ 5$ billion, or $7 \%$ of the total amount recoverable. This figure does not include punitive or other consequential damages nor does it include civil fines. These additions will make the civil liability fund even more inadequate.»

$112 \mathrm{El}$ acuerdo se encuentra entre otras páginas Web en:

http://www.tobaccofreekids.org/html/

http://www.house.gov/commerce/issues/tobacco/Tobacco.settlement.html.

http://stic.neu.edu/settlement/index.html.

113 Para el A Public Health Analysis of the Porposed Resolution, loc. cit., los contribuyentes absorberán el $30-40 \%$ del coste del acuerdo, ya que la deducción impositiva se 
5. Cuando se negociaba el preacuerdo, la industria tabaquera buscaba la protección contra todas las demandas presentes y futuras en su contra, mientras que la otra parte deseaba mantener el derecho de los particulares a demandar a la industria. El preacuerdo llegó a un término medio en el que los límites se colocaban en el derecho a demandar, sin que este derecho desaparezca. El preacuerdo, en aras a asegurar las futuras reclamaciones, establecía la creación de ciertos fondos que se extraerían de los pagos de la industria y que serían destinados al abono de indemnizaciones en el futuro. El volumen de estos fondos sería de 2 billones de dólares el primer año y se elevaría a 5 billones en el año nuevo (los 5 billones representan el $80 \%$ de la cantidad global disponible para indemnizaciones futuras). En contraprestación, se establecían una serie de restricciones en las demandas de reclamación de daños por la conducta de las Compañías tabacaleras en el pasado ${ }^{114}$. Algunas de las restricciones eran ${ }^{115}$ :

-Eliminar las indemnizaciones de daños punitivos.

-Eliminar las demandas bajo la forma de class action.

-Establecer una responsabilidad conjunta para todas las tabacaleras que formen parte del acuerdo.

- Limitar los demandados a los fabricantes o a sus agentes.

- Prohibir la prueba en el juicio de productos con riesgo reducido.

- Limitar los daños por los que se tiene derecho a indemnización.

Las opiniones contrarias plantearon que el preacuerdo aumentaba las barreras para entablar una demanda contra la industria de tal forma que la hacía caso imposible. Asimismo, el preacuerdo no establecía fondos suficientes para abonar indemnizaciones justas. La desaparición de los daños punitivos y los límites en las indemnizaciones harían casi imposible, para los futuros demandantes, el encontrar un abogado, ya que éste carecería de intereses económicos o todo el riesgo se trasladaría al demandante. El ahorro en costes para los demandantes, que había supuesto la interposición de demandas bajo la forma de la class action, también desaparecería ${ }^{116}$.

compensará con mayores impuestos. Estiman que la deducción de impuestos alcanzará los 4 billones anuales.

114 Según el informe A Public Health Analysis of the Porposed Resolution, loc. cit., bajo los términos del acuerdo la industria evitará unos 150-200 billones de dólares en responsabilidad al coste de 6-7 billones de dólares.

115 La lista completa de las restricciones que forman parte del acuerdo se recogen en el Título VIII, subpárrafo B del mismo.

116 A Public Health Analysis of the Porposed Resolution, loc. cit.: 
El preacuerdo establecía además la protección de la industria tabaquera eliminando todas las acciones presentes de los Attorneys General y otras similares entabladas por los Gobiernos, todas las demandas de terceros y todas las demandas entabladas por adicción a la nicotina ${ }^{117}$.

Una de las restricciones que más dudas plantea es la de las class actions existentes. Si el preacuerdo salda las class actions, entonces sus miembros tendrían derecho a alguna indemnización. Si el preacuerdo únicamente anulaba la orden de certificación del grupo y permitía que sus miembros entablasen demandas individualmente, es posible que entonces no tengan derecho a indemnización alguna. La cuestión no está clara, aunque la mayoría considera que el preacuerdo saldaría las class actions y que sus integrantes no podrían entablar demandas futuras de forma individual.

6. La entrega de los documentos que las tabacaleras habían elaborado durante los últimos treinta años también fue inicialmente considerada como un éxito de los Attorneys General. Sin embargo, una lectura de la medida muestra que el éxito era, por lo menos, relativo ${ }^{118}$. Es muy poco probable que la industria tabacalera, que todavía es vulnerable a sufrir ataques en el ámbito de lo penal por su comportamiento pasado y a demandas civiles en el extranjero, esté dispuesta a entregar los documentos más relevantes que puedan recoger pruebas incriminatorias. El

«The changes to the civil liability structure will also provide protections to the industry which will solidify its market and help guarantee future profitability. One of the primary reasons for having a civil liability system is to provide incentives for products manufacturers to behave honestly and produce safe products. The cost and risk attendant with litigation makes many corporations risk averse such that they strive to create safe products. A large jury verdict in a civil liability case can create shock waves throughout an industry. The changes to the civil liability system will eliminate these incentives.»

117 Ver Título VIII, subpárrafo A del acuerdo.

118 Summary of Settlement Provisions (Appendix VIII, p. 64):

«The settlement requires manufacturers to establish and maintain a centralized depository for documents "produced" in pending litigation. The documents will be available to Congress, state and federal agencies, and the public under certain conditions. The industry is permitted, however, to withhold any documents it considers to be "privileged" and any materials it considers to represent trade secrets. Materials regarding research on health, safety and less hazardous products also will be included, with the exception of legitimate trade secrets.

Upon settling the AG suits, the companies are permitted to rereview all documents claimed as privileged and create a new inventory of priviliged documents. Anyone wishing to challange the industry's assertion of privilege on trade secret must file a claim, which will be decided by a threejudge panel.» 
mecanismo para resolver las disputas sobre los documentos privilegiados es complicado y excesivamente largo ${ }^{119}$.

7. En agosto de 1997 tuvo lugar en Pekín la «X Conferencia Mundial sobre el Tabaco y la Salud», en la que se adoptó la siguiente resolución:

«The Conference recommends governments consider the international implications of tobacco control policies or settlements with the tobacco industry, to ensure that:

1. such measures do not contribute to an increase in the worldwide epidemic of tobacco-related death and disease;

2. the legal rights of those not party to any agreement or policy are fully protected;

3. such measures do not inhibit full public scrutiny of the past, present and future activities of the tobacco industry and;

4. that the tobacco industry pay the cost of damage caused by tobacco.»

Si el preacuerdo de junio de 1997 se hubiese aprobado estaría incumpliendo la resolución anterior. Primero, porque las tabacaleras no serían declaradas responsables por la totalidad de los daños causados por el tabaco. Segundo, porque los Estados Unidos se convertirían en un mercado mucho menos hospitalario de lo que ha sido hasta ahora y, esta situación probablemente incite a la industria del tabaco a establecer empresas fabricantes de tabaco fuera de sus fronteras para recuperar los beneficios pasados. Tercero, porque no cumple la segunda provisión al limitar los derechos legales de aquellos que no participaron en el acuerdo (por ejemplo, las demandas de particulares). Por último, tampoco se cumple la provisión cuarta porque el acuerdo no requiere que la industria entregue todos los documentos y toda la información que se halla en su poder.

119 AMERICAN CANCER SOCIETY, Official Position, Internet, http://www.tobacco.neu.edu/

La American Cancer Society ha recomendado unos cambios:

"Congress should ensure that both state and federal courts have jurisdiction to quickly resolve privilege claims. The burden should be on industry to demostrate why documents should not be disclosed.

Federal legislation regarding disclosure of industry documents should also explicity require the industry to release to the FDA all information including research and marketing data that is relevant to public health, safety and the development of less hazardous tobacco products.» 
8. Otras críticas que se han hecho al preacuerdo son:

-El preacuerdo se concentra casi de forma exclusiva en la Food and Drug Administration (FDA), en lugar de reconocer que hay otras agencias con jurisdicción sobre el tabaco. El preacuerdo ignora el hecho de que la mayoría de los progresos en el tema del tabaco se ha realizado en el nivel local y estatal. La FDA ya tiene jurisdicción sobre el tabaco y las provisiones del acuerdo, que no son ahora parte de las normativas de la FDA, podrían serlo sin que éste fuese necesario. Las provisiones sobre las fumadores pasivos del preacuerdo representan un paso atrás en el poder actual del Department of Labor.

-Existen medidas diseñadas para penalizar a la industria por incumplir o no alcanzar las reducciones en el número de menores fumadores. Estas medidas suponen un buen ejemplo de cómo las medidas técnicas del preacuerdo tienen impactos negativos importantes que no son evidentes a primera vista.

De hecho, la pena a imponer es demasiado pequeña para proveer de un incentivo económico efectivo para que la industria reduzca el consumo de tabaco en los jóvenes.

- En el preacuerdo hay una serie de medidas que incrementan las barreras para entrar en el mercado del tabaco. Esto promueve el comportamiento anticompetitivo y elimina cualquier incentivo para innovar en productos más seguros. Además, al cerrar el mercado, las nuevas compañías encontrarían que la competencia es más difícil. Esta situación garantizará la obtención de unos beneficios mayores que los actuales.

- Hay grupos que consideran que los esfuerzos descentralizados funcionan mejor en el control del tabaco, por lo que se oponen a cualquier medida que impida los controles locales ${ }^{120}$.

Preacuerdo de 23 de noviembre de 1998: Multistate Master Settlement Agreement (MSA)

El preacuerdo de 1997 llegó al Congreso en marzo de 1998 en forma de un proyecto de ley presentado por el Senador John McCain. El Commerce Committee elaboró una versión preliminar modificada el 18

120 Siegel, M.C.J., Hobart, R., Schoenarklin, S., Du Melle, F., Fisher, P., Preempetion in Tobacco Control: Review of an Emerging Public Health Problem, JAMA, 1997, pp. 858-863. 
de mayo ${ }^{121}$. El 8 de abril de 1998 el grupo Nabisco RJR abandonó el acuerdo al considerar que éste no refleja los términos aceptados el 20 de junio de 1997. Lo mismo hacen otras empresas tabaqueras importantes con lo que el 17 de junio de 1998 la versión preliminar presentaba ante el Congreso por el Commerce Committe se retira ${ }^{122}$.

Las tabacaleras habían llegado a acuerdos individuales con 4 estados (Mississippi, Florida, Minnesota y Texas), los cuales cuestionaron la necesidad de una acción del Congreso para solucionar el conflicto. Además, estos estados habían logrado llegar a acuerdos que compensan, casi en su totalidad, los costes de Medicaid junto con importantes medidas adicionales de sanidad pública; por ejemplo, la creación de programas antitabaco. Aunque no se puede extrapolar las normas de estos tres acuerdos, ya que cada uno presenta una situación de hecho y de derecho única, esta experiencia fue utilizada para iniciar las negociaciones con los Attorney General del resto del país. El 23 de noviembre de 1998 se alcanzó el Multistate Master Settlement Agreement entre 46 estados y las empresas tabaqueras más importantes ${ }^{123}$ por los gastos de Medicaid de los fumadores enfermos.

La diferencia principal del MSA con el preacuerdo de 1997 radica en que el MSA no requiere aprobación del Congreso. La naturaleza del MSA es la de un contrato entre las partes sancionado por los tribunales y no afecta, en principio, a cuestiones de salud pública. El MSA requiere que cada estado firmante someta el acuerdo al tribunal competente. De esta forma se evita el escollo de someter el acuerdo a la aprobación del Congreso. Además, debemos destacar que el acuerdo de 1998 no afecta a las class actions entabladas en los tribunales, ni a la normativa presente o futura de la FDA.

Las líneas maestras del MSA son:

1) Las tabacaleras firmantes se comprometen a abonar 206 billones de dólares a los estados firmantes por las enfermedades relacionadas con el tabaco ${ }^{124}$.

121 Bill S. 1415rs. Las líneas maestras del proyecto del Commerce Committee era: 1) la industria debía pagar 516 billones (en el preacuerdo de 1997 la cantidad era de 368,5 billones) durante 25 años para abonar los costes médicos causados por el consumo de tabaco; 2) el impuesto sobre el tabaco se eleva a 1,10 dólares por paquete; 3) mantiene la capacidad de la FDA para emitir normas sobre la industria del tabaco en forma distinta a la aceptada en el preacuerdo de 1997; 4) reduce la publicidad del tabaco.

122 El 17 de junio de 1998 el Senado rechazó el proyecto de ley por 57 votos en contra y 42 a favor.

123 Philip Morris, R.J. Reynolds Tobacco, Lorillard Tobacco y Brown \& Williamson.

${ }^{124}$ Los estados recibirían 12 billones durante los próximos 5 años, y el resto de los pagos se haría hasta el año 2025. 
2) La industria se compromete a patrocinar una campaña anti-tabaco de 1,5 billones de dólares.

3) Deben entregar los documentos conocidos como «Brown \& Williamson».

4) Tanto los estados firmantes como las empresas se comprometen a reducir el consumo de tabaco entre los menores. Parte del fondo económico que recibirán los estados deberá ser utilizado para cumplir con este compromiso.

5) Se imponen restricciones en la publicidad relacionada con el tabaco como, por ejemplo, la prohibición de dibujos animados, en especial los de Joe Camel.

6) Las tabacaleras ofrecen 5 billones de dólares a los agricultores relacionados con su industria, por la menor demanda como consecuencia del acuerdo.

7) Los estados se comprometen a retirar las 46 demandas planteadas contra la industria y a no entablar demandas futuras por costes sanitarios. No sólo se impide la interposición de demandas por conductas pasadas sino también algunas relacionadas con posibles conductas futuras de las tabacaleras y de empresas relacionadas. Se mantiene el derecho de los estados a entablar demandas por conductas criminales y también se establece una excepción, de forma expresa, para las cuestiones relacionadas con el sistema impositivo.

La ratificación del acuerdo alcanzado en noviembre de $1998^{125}$ no sólo afectaría a las tabacaleras, los estados y los fumadores (pasivos o activos). Otros sectores de la economía y de la cultura norteamericana también se verían afectados. Independientemente de si el acuerdo se aprueba de forma definitiva por el número suficiente de tribunales o no, lo que es importante es que a las tabacaleras les resultará muy difícil, si no imposible, volver a la situación anterior. Es decir, no parece posible que las tabacaleras vuelvan a presentar un frente común en los tribunales y se nieguen de forma sistemática a la negociación. Lo que se desprende es que la estrategia de la industria respecto a las demandas de los Attorney General es la negociación.

125 El 4 de febrero de 1999, la National Association of Attorney General informó que en 41 estados se había dado la aprobación al acuerdo. Ver gttp://www.naag.org/ consent.htm. 


\section{Recapitulación}

La industria del tabaco sigue teniendo dos frentes abiertos en su contra: las demandas entabladas por los particulares de forma individual y las class actions. El futuro en torno a ambas está por definir. A pesar de que, en la actualidad, los jurados son favorables a emitir un veredicto a favor de los demandantes, lo cierto es que suele ser revocado en apelación ${ }^{126}$. Sin embargo, las demandas continúan planteándose ante los tribunales y su número no sólo no se ha reducido con el paso del tiempo sino que continúa aumentando. El caballo de batalla se encuentra en la aceptación en juicio de los documentos Brown \& Williamson.

No nos parece probable que la situación descrita en las páginas precedentes pueda repetirse en el derecho continental o en el de Gran Bretaña. La existencia de daños punitivos, de juicio civiles con jurado, de «honorarios contingentes» para los abogados, de «class actions», y de otras medidas no existentes en Europa, hace que sea más fácil, en los Estados Unidos, recurrir ante el poder judicial para resolver ciertas contiendas. Es cierto que en Gran Bretaña han existido algunas demandas similares a las que existieron en la década de los ochenta en los Estados Unidos, pero su importancia es, hasta el momento, reducida. En España, dos han sido las demandas entabladas contra Tabacalera (en Barcelona y en Bilbao) y desconocemos cuál será la respuesta de los tribunales. 